\title{
Electron runaway in ASDEX Upgrade experiments of varying core temperature
}

\author{
O. Linder ${ }^{\oplus 1}$, $^{\dagger}$, G. Papp ${ }^{\oplus 1}$, E. Fable ${ }^{1}$, F. Jenko ${ }^{1}$, G. Pautasso ${ }^{1}$, the ASDEX \\ Upgrade Team $\ddagger$ and the EUROfusion MST1 Team $\S$ \\ ${ }^{1}$ Max-Planck-Institut für Plasmaphysik, 85748 Garching, Germany
}

(Received 13 January 2021; revised 29 March 2021; accepted 30 March 2021)

The formation of a substantial postdisruption runaway electron current in ASDEX Upgrade material injection experiments is determined by avalanche multiplication of a small seed population of runaway electrons. For the investigation of these scenarios, the runaway electron description of the coupled 1.5-D transport solvers ASTRA-STRAHL is amended by a fluid model describing electron runaway caused by the hot-tail mechanism. Applied in simulations of combined background plasma evolution, material injection and runaway electron generation in ASDEX Upgrade discharge \#33108, both the Dreicer and hot-tail mechanism for electron runaway produce only $\sim 3 \mathrm{kA}$ of runaway current. In colder plasmas with core electron temperatures $T_{\mathrm{e}, \mathrm{c}}$ below $9 \mathrm{keV}$, the postdisruption runaway current is predicted to be insensitive to the initial temperature, in agreement with experimental observations. Yet in hotter plasmas with $T_{\mathrm{e}, \mathrm{c}}$ above $10 \mathrm{keV}$, hot-tail runaway can be increased by up to an order of magnitude, contributing considerably to the total postdisruption runaway current. In ASDEX Upgrade high-temperature runaway experiments, however, no runaway current is observed at the end of the disruption, despite favourable conditions for both primary and secondary runaway.

Key words: fusion plasma, runaway electrons, plasma simulation

\section{Introduction}

In future current-carrying fusion devices, the formation of a substantial population of runaway electrons during the sudden loss of thermal confinement poses a significant threat to the integrity of the plasma vessel. Already in present-day devices, beams of lost runaway electrons are observed to damage plasma facing components, e.g. at JET (Matthews et al. 2016) or at Alcator C-Mod (Tinguely et al. 2018). However, in high-current devices, a larger runaway current is expected due to increased avalanche multiplication (Boozer 2019). As the total energy carried by a runaway beam grows quadratically with the runaway current (Martín-Solís et al. 2014), the threat to high-current fusion devices is amplified. Therefore, runaway electron generation has to be suppressed and potential disruptions mitigated (Breizman et al. 2019).

$\dagger$ Email address for correspondence: oliver.linder@ipp.mpg.de

\$ See author list of H. Meyer et al. 2019 Nucl. Fusion 59, 112014.

$\S$ See author list of B. Labit et al. 2019 Nucl. Fusion 59, 086020. 
Suppression of electron runaway may be achieved through massive material injection, as proposed for ITER (Lehnen et al. 2015). This scheme is currently being investigated across several machines using massive gas injection (MGI), e.g. at ASDEX Upgrade (AUG) (Pautasso et al. 2017, 2020) or TCV (Coda et al. 2019), and shattered pellet injection (SPI), e.g. at DIII-D (Commaux et al. 2010; Paz-Soldan et al. 2020) and JET (Reux et al. 2021). Given the unfavourable scaling of the runaway electron threat to future devices due to increased avalanche multiplication, experimental investigation is complemented by theoretical and computational studies to aid in extrapolation from present to future devices (Breizman et al. 2019).

Owing to the complexity of the runaway electron problem, different computational tools are used for the investigation of different aspects of electron runaway. The most accurate description is achieved by kinetic tools, such as the full- $f$ Fokker-Planck solver CODE (Stahl et al. 2016), where the runaway fluxes are determined through evolution of the momentum-space electron distribution. However, the simultaneous spatio-temporal evolution of the background plasma or impurities injected is challenging to calculate in these frameworks (Hoppe et al. 2021). For this purpose, 1-D transport codes such as ASTRA-STRAHL (Dux et al. 1999; Fable et al. 2013; Linder et al. 2020) or GO (Papp et al. 2013; Vallhagen et al. 2020) can be applied. Here, electron runaway is described through a fluid treatment, as a kinetic description greatly increases the computational cost. For a description of the 3-D spatio-temporal evolution of the magnetic field during disruptions, non-linear magnetohydrodynamic (MHD) codes such as JOREK (Bandaru et al. 2019) are used.

The recent development of sophisticated reduced kinetic models describing electron runaway due to momentum-space diffusion of thermal electrons (Hesslow et al. 2019b) and knock-on collisions of existing runaways with the thermal bulk (Hesslow et al. 2019a) has accelerated modelling efforts. Applied inside the transport code ASTRA-STRAHL, simulations of the spatio-temporal evolution of runaway electron population, background plasma and material injected have recently been found capable of describing AUG disruptions, as demonstrated modelling AUG discharge \#33108 (Linder et al. 2020).

In this work, we investigate runaway electron (seed) generation in AUG experiments of varying core temperature between 4 and $20 \mathrm{keV}$ by means of ASTRA-STRAHL simulations. For this purpose, we expand upon the findings by Linder et al. (2020), performing coupled simulations of background plasma evolution, material injection and electron runaway. As kinetic modelling using CODE suggests formation of a seed population of runaway electrons predominantly due to rapid cooling (Insulander Björk et al. 2020; Hoppe et al. 2021), the runaway electron generation models used in ASTRA-STRAHL are amended by a model by Smith \& Verwichte (2008) describing this effect. The toolkit ASTRA-STRAHL is then applied for the investigation of the (seed) runaway electron population in simulations of AUG discharge \#33108. Throughout the simulations performed, the preinjection on-axis electron temperature is varied between 4 and $20 \mathrm{keV}$, as SPI experiments in DIII-D suggest a growing seed runaway population as the electron temperature increases (Paz-Soldan et al. 2020). The simulation results obtained are compared against measurements of AUG disruption experiments.

This paper is organized as follows. A brief description of the model employed is provided in $\S 2$, with experimental aspects of AUG discharge \#33108 covered in $\S 3$. More details on both parts can be found in Linder et al. (2020). Simulations of runaway electron generation in the AUG discharge chosen are presented in $\S 4$. The impact of a variation of the preinjection on-axis electron temperature on the postdisruption runaway electron current calculated is discussed in $\S 5$. Finally, a conclusion is provided in $\S 6$. Additionally, a simplified model for the hot-tail runaway electron current density at the 
end of the thermal quench is presented in Appendix A; the impact of the average runaway electron velocity on the postdisruption runaway current is discussed in Appendix B.

\section{Model description}

The spatio-temporal evolution of the main tokamak plasma, material introduced through MGI, and runaway electrons generated in the process can be described by the coupled 1.5-D transport codes ASTRA (Fable et al. 2013) and STRAHL (Dux et al. 1999). The suitability of this toolkit for the simulation of runaway electron generation during MGI has recently been demonstrated by Linder et al. (2020). Building on the model presented, the capabilities of ASTRA-STRAHL are expanded to additionally consider electron runaway due to the hot-tail mechanism. Therefore, only a brief overview of ASTRA-STRAHL is given, with details described in Linder et al. (2020).

\subsection{The coupled transport codes ASTRA-STRAHL}

The evolution of the main plasma and impurity species introduced is calculated by ASTRA and STRAHL, respectively, following the macroscopic transport equation

$$
\frac{\partial Y}{\partial t}=\left(\frac{\partial V}{\partial \rho}\right)^{-1} \frac{\partial}{\partial \rho}\left(\frac{\partial V}{\partial \rho}\left\langle(\Delta \rho)^{2}\right\rangle\left\{D \frac{\partial Y}{\partial \rho}-v Y\right\}\right)+\sum_{j} S_{j}
$$

for a fluid quantity $Y$ in the presence of diffusion $D$, convection $v$ and sources $S_{j}$. The quantity $\rho$ denotes the toroidal flux-surface label, with $V$ being the flux-surface volume.

Inside ASTRA, the poloidal magnetic flux $\Psi$, both the electron temperature $T_{\mathrm{e}}$ and ion temperature $T_{\mathrm{i}}$ and the density $n_{\mathrm{RE}}$ of runaway electrons are evolved. In the case of electron heat transport, sources $S_{j}$ due to Ohmic heating, electron-to-ion heat transport and impurity radiation from STRAHL (line radiation and Bremsstrahlung) are taken into account throughout the entirety of the simulations and assumed to outweigh radial transport (Fehér et al. 2011) (confirmed by the simulations presented). Consequently, turbulent radial transport is neglected. The electron density $n_{\mathrm{e}}$ is calculated from quasi-neutrality, i.e. $n_{\mathrm{e}}(t)=n_{\mathrm{D}}+\sum_{k}\left\langle Z_{k}\right\rangle n_{k}(t)$ where $n_{\mathrm{D}}$ denotes the density of deuterium, the main plasma species. The densities $n_{k}$ and average charges $\left\langle Z_{k}\right\rangle$ of the impurities $k$ are evolved by STRAHL. The magnetic equilibrium is obtained from the ASTRA built-in 3-moment solver, applicable for circular discharges of MGI experiments in AUG (Pautasso et al. 2017, 2020).

The impurity densities $n_{k, i}$ are evolved by STRAHL for each charge state $i$ under consideration of electron impact ionization and recombination rates from ADAS (Summers 2004). Neutrals originating from a gas valve are deposited in the simulation domain just outside the last closed flux surface (LCFS) and propagate into the core plasma with thermal velocity $v_{k, 0}=v_{\text {th }}=\sqrt{T / m}, T$ and $m$ being the neutral impurity temperature and mass, respectively. The source strength $-\mathrm{d} N_{k} / \mathrm{d} t$ is determined from the continuity equation $\mathrm{d} N_{k} / \mathrm{d} t+v_{k, 0} N_{k} A_{\mathrm{v}}(t) / V_{\mathrm{v}}=0$ for a valve with particle inventory $N_{k}$, aperture size $A_{\mathrm{v}}(t)$ and volume $V_{\mathrm{v}}$. Impurity transport due to neoclassical processes is described by NEOART (Peeters 2000).

Following the injection of impurities, $(2,1)$ MHD modes and higher harmonics are triggered as the cold gas front reaches the flux surface with safety factor $q=2$ (Fable et al. 2016) at time $t_{q=2}$. As a result, the current density inside the $q=2$ surface is redistributed, which is achieved in the simulations by flattening the $q$-profile to $q=2$ under conservation of the total poloidal magnetic flux. During the breakup of the magnetic surfaces, the transport of ionized material and heat is greatly enhanced until closed flux surfaces have re-emerged. To mimic this effect inside ASTRA, additional transport coefficients of the 
form

$$
X_{\text {add }}(t)=X_{\text {add }}^{\max } \exp \left(-\frac{t-t_{q=2}}{\tau_{\text {add }}}\right) \Theta\left(t-t_{q=2}\right)
$$

are applied for both diffusive and convective transport inside the $q=2$ surface with $D_{\text {add }}^{\max }=100 \mathrm{~m}^{2} \mathrm{~s}^{-1}, v_{\text {add }}^{\max }=-1000 \mathrm{~m} \mathrm{~s}^{-1}, \chi_{\text {add }}^{\max }=100 \mathrm{~m}^{2} \mathrm{~s}^{-1}$ (Fehér et al. 2011) and $\tau_{\text {add }}=$ $1.0 \mathrm{~ms}$. The evolution of plasma parameters in ASTRA-STRAHL simulations applying this approach for discharge AUG \#33108 studied in this work has been compared in detail to experimental observations in the publication by Linder et al. (2020), where application of these coefficients was found necessary to reproduce the experimentally observed increase of the line-averaged electron density. Please note, that in this work, the additional transport coefficients are set to generic values (instead of a refined fit) as experimental observations are adequately described under a moderate variation of these coefficients by up to $50 \%$. In the simulations of varying preinjection on-axis electron temperature between 4 and 20 $\mathrm{keV}$ discussed in $\S 5$, transport coefficients of identical magnitude are prescribed, since the MHD modes triggered are largely current driven. As such, a (strong) dependence of the mode amplitude on predisruption temperature and pressure is not expected. A more detailed investigation on this subject is planned for future work.

The simulations presented in this work are carried out employing a radial grid of 401 points inside ASTRA, extending from the magnetic axis up to the LCFS. For STRAHL calculations, the grid is expanded to additionally include the scrape-off layer. Both minimum and constant time step in ASTRA and STRAHL, respectively, are set to $10^{-5}$ $\mathrm{ms}$ to resolve transient events. The suitability of these simulation settings was ensured by means of convergence scans of radial and temporal resolution in prestudy simulations.

\subsection{Runaway electron generation}

The process of electron runaway is described by reduced fluid models, providing sources $S_{j}$ for the evolution of the runaway electron density $n_{\mathrm{RE}}$ inside ASTRA ${ }^{1}$. Mechanisms for runaway electron generation considered in this work include hot-tail generation due to rapid cooling (see $\$ 2.2 .1$ ), Dreicer generation due to momentum-space diffusion of thermal electrons (see $\$ 2.2 .2$ ) and avalanche generation due to knock-on collision of existing runaway with thermal electrons (see $\S 2.2 .3$ ). Further mechanisms due to nuclear processes (Vallhagen et al. 2020) are not taken into account given AUG's non-nuclear environment. Feedback of the runaway electron population on the poloidal magnetic flux evolution $\Psi(t)$ is considered by adding the runaway electron current density to the total plasma current density under the assumption that runaway electrons propagate with a velocity $\left\langle v_{\mathrm{RE}}\right\rangle$ equal to the speed of light $c$, i.e. $\left\langle v_{\mathrm{RE}}\right\rangle=c$.

\subsubsection{Hot-tail generation}

In events of rapid plasma cooling, as in the case of tokamak disruptions, electron runaway may occur. Under these conditions, the high-energy tail of the electron energy distribution function equilibrates slower than the thermal bulk and may thus exceed the critical energy for runaway (Chiu et al. 1998; Harvey et al. 2000). The runaway electron population generated due to this process can be described by reduced fluid models, e.g. by the work of Smith \& Verwichte (2008) and Fehér et al. (2011). However, compared with kinetic simulations with the full- $f$ continuum Fokker-Planck solver CODE (Stahl et al. 2016), these reduced models are found to underestimate the hot-tail density by up to an order of magnitude as the impact of the electric field on the underlying electron

\footnotetext{
${ }^{1}$ The runaway electron generation models discussed are implemented as a standalone Fortran module, available at https://github.com/o-linder/runawayelectrongeneration.
} 
distribution function is not taken into account by these models (Breizman et al. 2019; Harvey et al. 2019). Simultaneously, the computational cost of kinetic solvers renders application in transport simulations impractical. Therefore, cheaper and more accurate models are currently being developed by Svenningsson (2020), which, however, are not available yet for practical applications with varying effective plasma charge $Z_{\text {eff }}$. For this reason, the model by Smith \& Verwichte (2008) is applied in this work for the calculation of the hot-tail runaway electron population. Note, that in a recent validation of this model by Petrov, Parks \& Harvey (2021) with the Fokker-Planck solver CQL3D, an additional $Z_{\text {eff }}$-dependent factor of order unity was proposed for the definition of the critical velocity.

According to the model by Smith \& Verwichte (2008), the hot-tail runaway electron density $n_{\text {hot }}$ at time $t$ is obtained from the velocity-space integral across the runaway region as

$$
n_{\mathrm{hot}}(t)=\frac{4 n_{\mathrm{e}, 0}}{\sqrt{\pi} v_{\mathrm{th}, 0}^{3}} \int_{v_{\mathrm{c}}(t)}^{\infty}\left(v^{2}-v_{\mathrm{c}}(t)^{2}\right) \exp \left(-\left[\frac{v^{3}}{v_{\mathrm{th}, 0}^{3}}+3 \tau(t)\right]^{2 / 3}\right) \mathrm{d} v,
$$

where $v_{\text {th }}$ denotes the thermal velocity, $v_{\text {th }}^{2}=2 T_{\mathrm{e}} / m_{\mathrm{e}}$, and $v_{\mathrm{c}}$ the critical velocity for electron runaway, $v_{\mathrm{c}}^{2}=e^{3} n_{\mathrm{e}} \ln \Lambda / 4 \pi \varepsilon_{0}^{2} m_{\mathrm{e}} E_{\|}$with $\ln \Lambda$ being the Coulomb logarithm for thermal-thermal collisions, i.e. $\ln \Lambda=16.1-0.5 \log \left(n_{\mathrm{e}} / 10^{19} \mathrm{~m}^{-3}\right)+\log \left(T_{\mathrm{e}} / \mathrm{keV}\right)$. The constants $m_{\mathrm{e}}, e$ and $\varepsilon_{0}$ denote, respectively, the electron mass, the elementary charge and the vacuum permittivity. Quantities evaluated at the onset of rapid cooling are denoted by indices ' 0 '. The parameter $\tau(t)$ is a normalized time, i.e. $\tau(t)=v_{0} \int_{t_{0}}^{t} n_{\mathrm{e}}(\tilde{t}) \mathrm{d} \tilde{t} / n_{\mathrm{e}, 0}$, with the thermal-thermal collision frequency $v=n_{\mathrm{e}} e^{4} \ln \Lambda / 4 \pi \varepsilon_{0}^{2} m_{\mathrm{e}}^{2} v_{\text {th }}^{3}$.

The expression (2.3) introduced by Smith \& Verwichte (2008) for the hot-tail density assumes an instantaneous drop of the electron temperature from $T_{\mathrm{e}, 0}$ to the final temperature $T_{\mathrm{e}, \text { fin }}$. However, motivated by an exponential decay of the temperature on time scales $t_{\text {dec }}$ due to plasma cooling (Smith \& Verwichte 2008), the hot-tail density evolution under assumption of an exponential decay of the temperature, i.e.

$$
T_{\mathrm{e}}(t)=\left(T_{\mathrm{e}, 0}-T_{\mathrm{e}, \mathrm{fin}}\right) \exp \left(-\frac{t-t_{0}}{t_{\mathrm{dec}}}\right)+T_{\mathrm{e}, \mathrm{fin}},
$$

can be described by modifying the expression for $\tau(t)$. In the work by Smith \& Verwichte (2008), the temporal evolution of this parameter is obtained through numerical integration of a high moment of the kinetic equation for a two-component distribution function. In the case of an exponential electron density evolution, the numerical solution obtained for $\tau(t)$ is well approximated by $\tau(t)=v_{0}\left(t-t_{0}-t_{\mathrm{dec}}\right) \Theta\left(t-t_{0}-t_{\mathrm{dec}}\right) n_{\mathrm{e}, \text { fin }} / n_{\mathrm{e}, 0}$ for $t-t_{0}>3 t_{\mathrm{dec}}$ (Smith \& Verwichte 2008). However, to describe $\tau(t)$ more accurately during the initial phase of rapid cooling, an alternative expression is introduced and used throughout this work

$$
\tau(t)=v_{0} \frac{n_{\mathrm{e}, \mathrm{fin}}}{n_{\mathrm{e}, 0}}\left\{\begin{array}{ll}
\frac{\left(t-t_{0}\right)^{2}}{4 t_{\mathrm{dec}}}, & t-t_{0}<2 t_{\mathrm{dec}} \\
\left(t-t_{0}-t_{\mathrm{dec}}\right), & t-t_{0} \geq 2 t_{\mathrm{dec}}
\end{array} .\right.
$$

For evaluation of the hot-tail density, a closed form of expression (2.3) cannot be provided, necessitating numerical integration. Inside ASTRA, the integral is evaluated using Kepler's rule as the integrand falls off monotonically and sufficiently fast for $v \rightarrow \infty$. The hot-tail runaway electron density $n_{\text {hot }}$ obtained can be used inside ASTRA directly for subsequent calculations of the runaway electron current density and secondary runaway generation, eliminating the necessity to evaluate the macroscopic transport equation (2.1) for the hot-tail population. However, importantly, evaluation of the instantaneous hot-tail 
population through (2.3) requires characterization of parameters at onset and end of the thermal quench, being the time $t_{0}$ of the onset of the thermal quench, the electron temperature $T_{\mathrm{e}}\left(t_{0}\right)$, the temperature decay time scale $t_{\mathrm{dec}}$, as well as the electron density at onset and end of the thermal quench, i.e. $n_{\mathrm{e}}\left(t_{0}\right)$ and $n_{\mathrm{e}}\left(t_{\mathrm{fin}}\right)$. As onset and end of the thermal quench cannot be determined during a simulation, the required parameters are calculated in postsimulation analysis (see § 2.3 ) and applied in a subsequent simulation for the calculation of the hot-tail population. Convergence of the parameters obtained has to be assessed and simulations repeated if convergence is not met. Note, that in the simulations presented in this work, one iteration to determine the thermal quench parameters was sufficient to achieve an averaged iteration accuracy of less than $1 \%$ across all grid points, being in all cases less than $5 \%$.

\subsubsection{Dreicer generation}

The process of electron runaway due to momentum-space diffusion of thermal electrons in the presence of partially ionized mid- to high- $Z$ impurities cannot be described by analytical reduced fluid models, as a result of the complicated energy dependence of collision frequencies at near-thermal energies (Hesslow et al. 2019b). Analytical expressions under consideration of fully ionized impurities only (Connor \& Hastie 1975) have been demonstrated to overestimate electron runaway under certain conditions (Hesslow et al. 2019b). Applied in transport simulations of runaway electron generation during MGI, a noticeably increased seed population in contrast to experimental observations is obtained (Linder et al. 2020). Therefore, instead of reduced fluid models, a neural network model for the calculation of Dreicer growth rates (Hesslow et al. 2019b) is utilized in this work.

The neural network by Hesslow et al. (2019b) is based on simulations of CODE. Training of the network was performed with argon and neon impurities, generalized for application to other species using eight input parameters $x$. The Dreicer source rate $S_{\mathrm{D}}$ is thus obtained through evaluation of

$$
\begin{gathered}
S_{\mathrm{D}}=v n_{\mathrm{e}} \exp \left(\mathcal{F}\left(\boldsymbol{W}_{5}, \mathcal{F}\left(\boldsymbol{W}_{4}, \mathcal{F}\left(\boldsymbol{W}_{3}, \mathcal{F}\left(\boldsymbol{W}_{2}, \mathcal{F}\left(\boldsymbol{W}_{1}, \boldsymbol{x}, \boldsymbol{b}_{1}\right), \boldsymbol{b}_{2}\right), \boldsymbol{b}_{3}\right), \boldsymbol{b}_{4}\right), b_{5}\right)\right), \\
\mathcal{F}(W, x, b)=\tanh (W x+b),
\end{gathered}
$$

with weights $\boldsymbol{W}_{i}$ and biases $\boldsymbol{b}_{i}$ (see Hesslow et al. (2019b) for details).

\subsubsection{Avalanche generation}

The generation of secondary runaway electrons due to knock-on collisions of existing runaways with thermal electrons in the presence of partially ionized impurities can be described through a reduced fluid model by Hesslow et al. (2019a). The avalanche source rate $S_{\text {av }}$ is calculated from

$$
S_{\mathrm{av}}=n_{\mathrm{RE}} \frac{e}{m_{\mathrm{e}} c \ln \Lambda_{\mathrm{c}}} \frac{n_{\mathrm{e}}^{\text {tot }}}{n_{\mathrm{e}}} \frac{E_{\|}-E_{\mathrm{c}}^{\mathrm{eff}}}{\sqrt{4+\bar{v}_{\text {slow }}\left(p_{\star}\right) \bar{\nu}_{\text {defl }}\left(p_{\star}\right)}},
$$

with the relativistic Coulomb logarithm $\ln \Lambda_{\mathrm{c}}=\ln \Lambda-0.5 \ln \left(T / m_{\mathrm{e}} c^{2}\right)$. The total electron density $n_{\mathrm{e}}^{\text {tot }}$ comprises both free plasma electrons $n_{\mathrm{e}}$ and electrons bound to impurity ions. In the presence of partially ionized impurities, the critical electric field $E_{\mathrm{c}}=$ $n_{\mathrm{e}} e^{3} \ln \Lambda_{c} / 4 \pi \varepsilon_{0}^{2} m_{\mathrm{e}} c^{2}$ for runaway is increased, the effect of which is described by the effective critical electric field $E_{\mathrm{c}}^{\text {eff }}$ defined in Hesslow et al. (2018b). Expressions for the slowing-down frequency $\bar{v}_{\text {slow }}$ and for the generalized deflection frequency $\bar{v}_{\text {deff }}$ are found in Hesslow et al. (2018a) and Hesslow et al. (2018b). Noticeably, the effective critical 
(a)

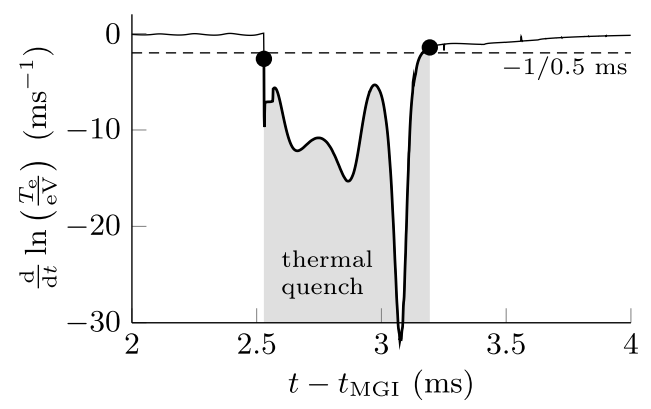

(b)

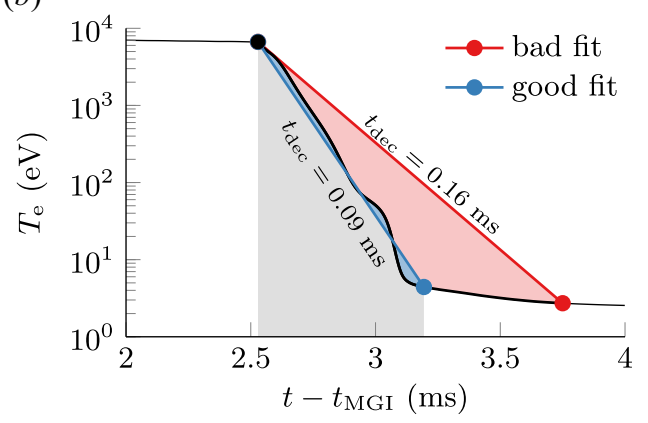

FIGURE 1. The occurrence of a thermal quench is determined from the electron temperature evolution starting at the onset of MGI at $t_{\text {MGI. }}$. $(a)$ The temporal derivative of the logarithmic temperature falling below a threshold of $-1 / 0.5$ ms marks the onset of the quench. $(b)$ The end is obtained from an exponential fit of the electron temperature, illustrated for both a suitable (blue) and a poor (red) choice of the decay time scale $t_{\mathrm{dec}}$.

momentum $p_{\star}$ depends on both frequencies through $p_{\star}=\sqrt[4]{\bar{\nu}_{\text {slow }}\left(p_{\star}\right) \bar{\nu}_{\text {deff }}\left(p_{\star}\right)} / \sqrt{E_{\|} / E_{\mathrm{c}}}$, thus requiring numerical evaluation of these parameters.

\subsection{Determining thermal quench parameters}

The calculation of characteristic quantities of the thermal quench for the evaluation of the hot-tail runaway population during the thermal quench (see $\$ 2.2 .1$ ) is performed in postsimulation analysis. Onset $t_{0}$ and end $t_{\text {fin }}$ of the thermal quench are determined from the electron temperature evolution. The required values for both the electron temperature and density are then obtained through evaluation of these quantities at $t_{0}$ and $t_{\text {fin }}$, respectively.

The time $t_{0}$ of the onset of the thermal quench is defined as the time when the instantaneous logarithmic temperature change $\mathrm{d} \ln \left(T_{\mathrm{e}}(t) / \mathrm{eV}\right) / \mathrm{d} t$ falls below a threshold value $-1 / \tilde{t}_{\mathrm{dec}}=-1 / 0.5 \mathrm{~ms}$, with $\tilde{t}_{\mathrm{dec}}$ being the instantaneous temperature decay time (see figure $1 a$ ). Both the end $t_{\text {fin }}$ of the thermal quench and the temperature decay time scale $t_{\mathrm{dec}}$ are determined through a linear fit $\ln \tilde{T}_{\mathrm{e}}(t)$ of the logarithmic electron temperature evolution (see figure $1 b$ ). Under the assumption $T_{\mathrm{e}}\left(t_{0}\right) \gg T_{\mathrm{e}}\left(t_{\text {fin }}\right)$, the ansatz for $T_{\mathrm{e}}(t)$ of (2.4) can be reduced to $\ln \left(T_{\mathrm{e}}(t) / T_{\mathrm{e}}\left(t_{0}\right)\right)=-\left(t-t_{0}\right) / t_{\mathrm{dec}}$, thus yielding the time scale $t_{\mathrm{dec}}$. The end of the thermal quench is defined as the last time point where $\tilde{T}_{\mathrm{e}}\left(t_{\text {fin }}\right)=T_{\mathrm{e}}\left(t_{\mathrm{fin}}\right)$, i.e. before the fit falls off below the actual temperature. The quality of the fit is evaluated for $t \in\left[t_{0}, t_{\text {fin }}\right]$.

\section{ASDEX Upgrade runaway electron experiments}

\subsection{Reference scenario}

Simulations of runaway electron generation are performed for artificially disrupted ASDEX Upgrade experiments through MGI (Pautasso et al. 2017, 2020). The plasma parameters chosen in this work are based on ASDEX Upgrade discharge \#33108 (for details see Linder et al. 2020). In this experiment, argon (Ar) was injected at $t_{\text {inj }}=1.0 \mathrm{~s}$ after breakdown from a gas valve of volume $100 \mathrm{~cm}^{3}$ and initial Ar pressure of $0.73 \mathrm{bar}$ into an L-mode limiter plasma with low average electron density of $\left\langle n_{\mathrm{e}}\right\rangle=2.8 \times 10^{19} \mathrm{~m}^{-3}$ and high peaked electron temperature of $T_{\mathrm{e}}(\rho=0)=9.3 \mathrm{keV}$ at the magnetic axis. A peaked temperature profile is achieved through application of $2.6 \mathrm{MW}$ of on-axis electron cyclotron resonance heating (ECRH) during the last $0.1 \mathrm{~s}$ prior to MGI. As a result of 


$\begin{array}{lcc}\text { Quantity } & \text { AUG \#33108 } & \text { Similar shots } \\ I_{\mathrm{p}, 0}(\mathrm{MA}) & 0.76 & 0.60-0.90 \\ p_{\mathrm{Ar}}(\mathrm{bar}) & 0.73 & 0.60-0.85 \\ B_{\text {tor }}(\mathrm{T}) & 2.50 & 2.30-2.70 \\ q_{95} & 3.79 & 3.50-4.10\end{array}$

TABLE 1. Characteristic parameters for runaway electron experiments in AUG, being the predisruptive plasma current $I_{\mathrm{p}, 0}$, the valve Ar pressure $p_{\mathrm{Ar}}$, the toroidal magnetic field $B_{\text {tor }}$ and the edge safety factor $q_{95}$. Values for the reference discharge AUG \#33108 are given, as well as criteria for selecting similar shots from all AUG runaway electron experiments performed.

Ar injection, the plasma stored energy is removed through impurity radiation and the plasma current decreases from initially $763 \mathrm{kA}$ down to $225 \mathrm{kA}$, carried by relativistic electrons. Additional characteristic parameters of AUG \#33108, as well as criteria for selecting similar runaway electron shots for analysis in $\S 5.5$, are listed in table 1 .

\subsection{Gaussian process regression for experimental fitting}

Reconstruction of experimental profiles often requires fitting of measured data. Application of a probabilistic approach under consideration of uncertainties allows a reliable estimate of experimental quantities. Therefore, Gaussian process regression (GPR) techniques are employed in this work through application of a toolset by Ho et al. (2019), based on work by Chilenski et al. (2015). Using these tools, reconstruction of preinjection electron temperature profiles $T_{\mathrm{e}}\left(t_{\mathrm{inj}}, \rho\right)$ from electron cyclotron emission (ECE) and Thomson scattering (TS) measurements is performed in $\S 5.1$ for the entire plasma radius and in $\S 5.5$ for a better reconstruction of the on-axis value $T_{\mathrm{e}}\left(t_{\mathrm{inj}}, 0\right)$. An estimation of the experimentally measured runaway electron current as a function of $T_{\mathrm{e}}\left(t_{\mathrm{inj}}, 0\right)$ is also performed using GPR.

Applying Bayesian probability theory, robust reconstruction of these profiles, as well as of associated gradients and uncertainties, is performed from covariance functions $k\left(x, x^{\prime}\right)$ utilizing normally distributed weights. Where stated in this work, profile estimation through GPR is performed using a rational quadratic covariance function

$$
k\left(x, x^{\prime}\right)=\sigma^{2}\left(1+\frac{\left(x-x^{\prime}\right)^{2}}{2 \alpha l^{2}}\right)^{\alpha}
$$

with variance $\sigma^{2}$ and characteristic length scale $l$. The hyperparameter $\alpha$ describes length scale mixing. When simpler estimates are sufficient, plasma profiles are instead reconstructed using an mtanh function (Schneider 2012).

\section{Electron runaway in ASDEX Upgrade \#33108}

Coupled transport simulations of Ar injection, background plasma evolution and runaway electron generation are performed with ASTRA-STRAHL for AUG discharge \#33108. The evolution of the Ar-induced disruption throughout the simulation is described in $\S 4.1$. The generation of a seed population is discussed in $\S 4.2$, whereas the avalanche multiplication following is covered in $\S 4.3$. The spatio-temporal evolution of the runaway electron current density contributions and the Ohmic current density is illustrated, in 
addition to this paper, in a supplementary movie available at https://doi.org/10.1017/ S0022377821000416.

\subsection{Simulation of thermal and current quench}

Simulating AUG discharge \#33108, the impurities injected reach the LCFS at $t=1.0 \mathrm{~ms}$ after the valve trigger. Note that in this section, the time $t$ is given with respect to the time $t_{\text {inj }}$ of the start of material injection. The cold gas front propagates further into the central plasma and in the process locally cools down the plasma through strong impurity radiation. As a result, the Ohmic current (with density $j_{\Omega}$ ) contracts inwards where the plasma temperature has not collapsed yet (see supplementary movie). Eventually, strong current density gradients $\mathrm{d} j_{\Omega} / \mathrm{d} \rho$ at the $q=2$ surface at $\rho=0.7$ excite $(m, n)=(2,1)$ MHD modes and higher harmonics, thus causing rapid redistribution of heat and material inside the $q=2$ surface. In the process, the remaining plasma stored energy is dissipated globally through impurity radiation on a sub-ms time scale, decreasing the electron temperature and therefore also the plasma conductivity. Following the law of induction, strong electric fields are generated. In this environment, a seed population of runaway electrons is created due to both the hot-tail and Dreicer mechanisms. During the slower decay of the residual Ohmic current, the runaway seed population is amplified by the avalanche mechanism, establishing a significant runaway electron current at the end of the current quench, being $333 \mathrm{kA}$ in the simulation.

\subsection{The runaway seed population}

Seed runaway electrons are generated due to the hot-tail and Dreicer mechanisms until the end of the thermal quench in the simulations performed. In the case of Dreicer generation, runaway occurs primarily in the vicinity of the $q=2$ surface at around $\rho=0.7$ prior to the thermal quench (see figure 2). As the material injected begins to propagate into the plasma centre, cooling it down in the process, the Ohmic current contracts inwards to locations where the temperature has not collapsed yet. As a result, a high Ohmic current density is located in front of the cold gas, growing in magnitude as the material propagates inwards further (see supplementary movie). The maximum Ohmic current density is observed in the vicinity of the $q=2$ surface. As the cold gas front reaches this location, $(2,1)$ MHD modes are triggered. In the process, the current density is flattened inside the $q=2$ surface. As follows from the relation

$$
\frac{E_{\|}}{E_{\mathrm{D}}}=\frac{m_{\mathrm{e}} v}{n_{\mathrm{e}} \mathrm{e}^{2}} j_{\Omega} \frac{4 \pi \varepsilon_{0}^{2} T_{\mathrm{e}}}{n_{\mathrm{e}} e^{3} \ln \Lambda}=\frac{\sqrt{m_{\mathrm{e}}}}{e n_{\mathrm{e}} \sqrt{8 T_{\mathrm{e}}}} j_{\Omega},
$$

strong electric fields $E_{\|}$normalized to the Dreicer electric field $E_{\mathrm{D}}=n_{\mathrm{e}} e^{3} \ln \Lambda / 4 \pi \varepsilon_{0}^{2} T_{\mathrm{e}}$ are induced at locations of high Ohmic current density $j_{\Omega}$, amounting to around $5 \% E_{\mathrm{D}}$ in the vicinity of the $q=2$ surface. With the generation of Dreicer runaway electrons being, in a simple picture, exponentially sensitive to $-E_{\mathrm{D}} / E_{\|}$(Connor \& Hastie 1975), a seed population of up to $18 \mathrm{kA} \mathrm{m}^{-2}$ is established in the vicinity of the $q=2$ surface.

During the thermal quench following, the electric field inside the $q=2$ surface increases up to $\sim 3 \% E_{\mathrm{D}}$ as the rapidly decaying Ohmic current is distributed inside the $q=2$ surface more evenly. However, due to the aforementioned exponential sensitivity, only a small population of additional runaway electrons is generated. Until the end of the thermal quench, the Dreicer mechanism produces a runaway current of $1.1 \mathrm{kA}$, constituting only around $0.3 \%$ of the total postdisruption runaway current. Consequently, the Dreicer mechanism is relevant only for establishing a small seed population of runaways. 


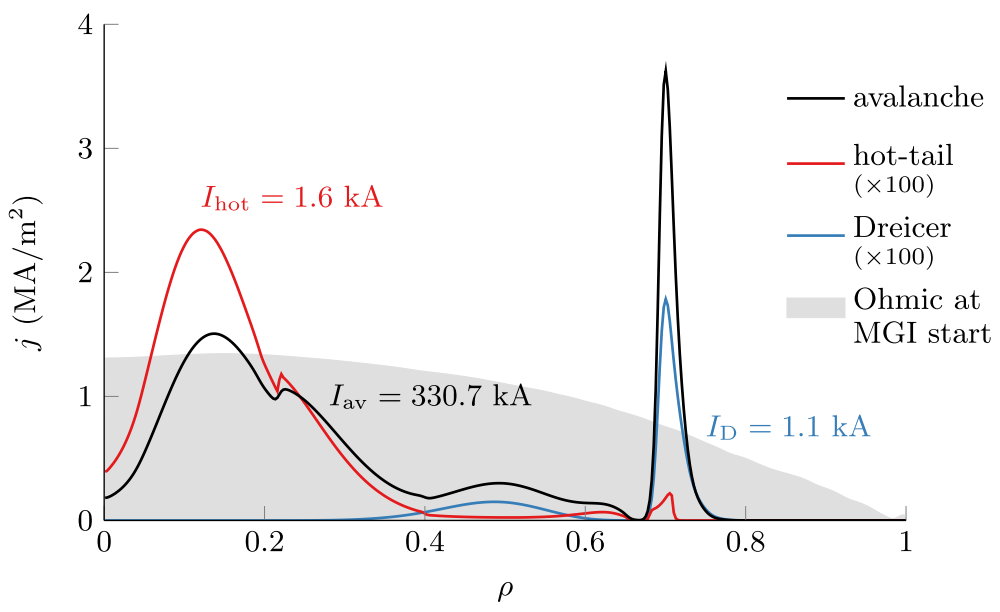

FIGURE 2. Radial distribution of the postdisruption runaway electron current densities $j$ at the end of the current quench in simulations of AUG \#33108, generated by the avalanche mechanism (black), by the hot-tail mechanism (red) and by the Dreicer mechanism (blue). The runaway electron current densities are compared against the Ohmic current density $j_{\Omega}$ at the start of MGI. Note, that the current densities of the hot-tail and Dreicer mechanism generated seed populations shown are multiplied by a factor of $\times 100$ given their small magnitude compared to the avalanche generated runaway current density. Additionally, the current $I$ carried by each population is shown next to the corresponding current density profile. The spatio-temporal evolution of the runaway electron current density is additionally shown in a supplementary movie of this figure.

The hot-tail mechanism for the generation of runaways becomes important during rapid decrease of the electron temperature. Prior to the thermal quench during the inward propagation of the cold gas front, these conditions are not met. Only with the onset of the thermal quench, a noticeable population of hot-tail runaways is created inside the $q=2$ surface (see figure 2). Importantly, significant generation of more than $1 \mathrm{kA} \mathrm{m}^{-2}$ of hot-tail current density occurs predominantly inside $\rho=0.4$, i.e. in the region where on-axis ECRH was applied prior to MGI. At the end of the thermal collapse, a total hot-tail current of $1.6 \mathrm{kA}$ is obtained, being around $0.5 \%$ of the postdisruption runaway current. Consequently, the hot-tail mechanism also provides only a small seed population of runaways in AUG \#33108.

The largest hot-tail current density max $j_{\text {hot }}$ is observed off-axis at $\rho=0.12$, while the on-axis current density amounts to only around $\max \left(j_{\text {hot }}\right) / 6$ despite a larger prequench temperature. This seemingly contradictory behaviour can be understood by evaluating the dominant contributions of the hot-tail model of (2.3), thus obtaining the simplified expression (see (A 9) of Appendix A)

$$
n_{\mathrm{hot}}^{\text {simple }}\left(t_{\text {fin }}\right)=\frac{2 n_{\mathrm{e}, 0}}{\sqrt{\pi}} \exp \left(-4\left\{\tilde{v} \ln \Lambda\left(t_{0}\right) \frac{n_{\mathrm{e}, \mathrm{fin}} t_{\mathrm{dec}}}{T_{\mathrm{e}, 0}^{3 / 2}}\right\}^{2 / 3}\right) .
$$

Hence, in a simple estimate, the postquench hot-tail density is exponentially sensitive to the prequench electron temperature $T_{\mathrm{e}, 0}$, to the decay time scale $t_{\mathrm{dec}}$, and to the postquench electron density $n_{\mathrm{e}, \text { fin }}$. Analysing the radial distribution of these quantities for AUG \#33108 (see figure $3 a$ ), the decay time is observed to be uniformly around $t_{\mathrm{dec}} \sim 0.1 \mathrm{~ms}$. Therefore, the hot-tail population is predominantly determined by the ratio $n_{\mathrm{e}, \text { fin }}^{2 / 3} / T_{\mathrm{e}, 0}$. Inside $\rho=0.4$, 
(a)

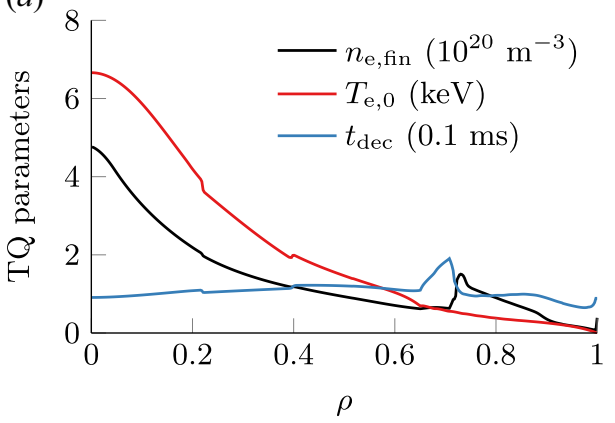

(b)

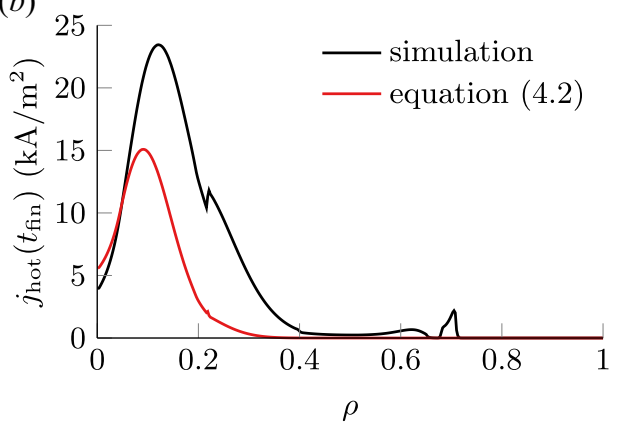

FIGURE 3. For simulations of AUG \#33108, $(a)$ parameters of the thermal quench (TQ), i.e. electron density at the end (black), electron temperature at the onset (red) as well as the temperature decay time scale (blue). (b) The postquench hot-tail current density obtained through simulations (black) is compared against an analytical estimate from (4.2) (red).

this ratio decreases as the electron temperature peaks due to preinjection ECRH. With higher prequench temperature, as well as due to inward impurity propagation, the impurity contributed free electron density postquench is increased as well, peaking close to the magnetic axis, and thus increasing the ratio $n_{\mathrm{e}, \text { fin }}^{2 / 3} / T_{\mathrm{e}, 0}$ close to the magnetic axis. As a result, the largest hot-tail population is observed off-axis.

The simple analysis following (4.2) is capable of reproducing the general trend of the hot-tail density obtained through evaluating the full expression (2.3) in simulations (see figure $3 b$ ). Consequently, the dependencies discussed are also valid for the complete model. It should be noted that the simplified model underestimates the hot-tail density especially in the outer half-radius. This behaviour occurs because factors appearing in a more general model (see (A 7)) were simplified based on AUG disruption parameters of the central plasma. Application of the more general simplified model of (A 7) yields an estimate of the hot-tail density larger than observed in simulations.

Following this analysis, the postquench hot-tail population observed is strongly influenced by both the prequench electron temperature, as well as by impurity deposition and propagation. A reduction of the hot-tail seed population can consequently be achieved by reducing the plasma temperature, slowing down the thermal quench, or depositing impurities predominantly in regions of highest temperatures.

\subsection{Runaway electron multiplication}

The vast majority of the runaway current observed in simulations of AUG \#33108, being $331 \mathrm{kA}$ or $99.1 \%$ of the postdisruption runaway current, originates from secondary runaway electrons (see figure 2), generated during knock-on collisions of thermal electrons with existing runaways from the small seed population. The radial distribution of the avalanche generated runaway current density is thus a scaled-up superposition of the seed populations. Consequently, the postdisruption runaway population is located primarily in the vicinity of the $q=2$ surface, as well as close to the magnetic axis at $\rho \sim 0.1$. Due to diffusion of the electric field during the current quench, the postdisruption runaway current density exceeds the predisruption Ohmic current density at these locations. As a result of impurity redistribution during the disruption and the associated impact on the evolution of the residual Ohmic current density, favourable conditions for avalanche multiplication exist predominantly close to, but inside the $q=2$ surface. Therefore, 


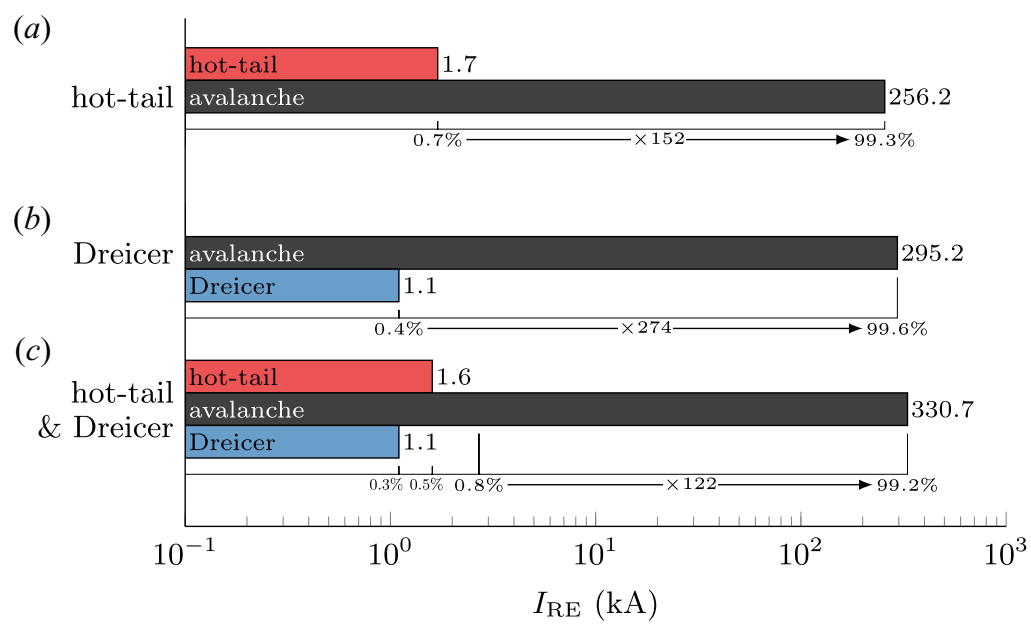

FIGURE 4. Comparison of the postdisruption runaway current contributions $I_{\mathrm{RE}}$ from seed and avalanche mechanisms in simulations of AUG \#33108 utilizing selected source mechanisms, being (1) only the hot-tail mechanism (a), (2) only the Dreicer mechanism (b), and (3) both the hot-tail and the Dreicer mechanism $(c)$. Both the absolute runaway currents, as well as the relative strength of each generation mechanism are specified. Additionally, the avalanche multiplication factor for each simulation is listed.

avalanche multiplication of the Dreicer generated seed population is stronger than for the hot-tail seed.

The importance of the avalanche mechanism for electron runaway in AUG \#33108 has also been observed by Insulander Björk et al. (2020) in simulations with the full- $f$ solver CODE. In their work, however, the small seed population was determined to consist almost entirely of hot-tail generated runaways with virtually no contribution from the Dreicer mechanism. Thus, to assess the impact of the individual source mechanisms on runaway multiplication in this framework, simulations of AUG \#33108 are repeated with only one of the primary generation mechanisms enabled.

In simulations considering either only the hot-tail or the Dreicer mechanism as a source for primary runaways, the seed population is reduced to $1.7 \mathrm{kA}(-37 \%)$ and $1.1 \mathrm{kA}$ $(-59 \%)$, respectively, compared with a seed population of $2.7 \mathrm{kA}$ obtained in the case of employing both mechanisms (see figure 4). Yet, the postdisruption runaway current obtained in both cases is not reduced proportionally, being $258 \mathrm{kA}(-23 \%)$ and 296 kA $(-11 \%)$, respectively. As the residual Ohmic current decays at similar time scales independent of the seed mechanisms employed, the postdisruption runaway current is thus determined by the avalanche multiplication time and seed population. Given the more favourable conditions for avalanche multiplication in the vicinity of the $q=2$ surface as discussed above, the smaller Dreicer generated runaway seed produces a larger secondary population than in the case utilizing only a hot-tail seed. Importantly, a comparable postdisruption runaway current is obtained in all three cases. Therefore, based on the simulations presented, the exact composition of the primary runaway seed seems to be of secondary importance in the case of AUG \#33108, as avalanche generation during the current quench dominates the dynamics. 


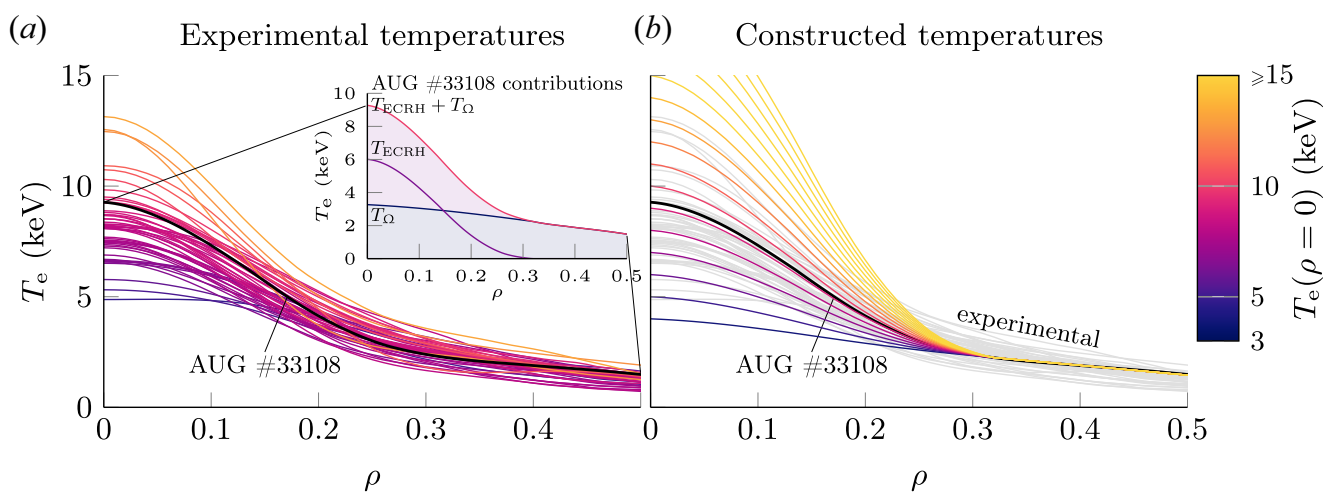

FIGURE 5. (a) Electron temperature profiles of AUG disruption experiments similar to discharge \#33108 (see table 1), constructed by GPR using ECE and TS measurement from the last $50 \mathrm{~ms}$ prior to MGI. The temperature profile of AUG \#33108 can be decomposed into a contribution $T_{\Omega}$ due to Ohmic heating and into a localized contribution $T_{\mathrm{ECRH}}$ due to on-axis ECRH. (b) Electron temperature profiles for the scan presented in $\S 5$ are constructed by using the profile of AUG \#33108 and scaling the ECRH contribution $T_{\mathrm{ECRH}}$, thus assuming application of varying amounts of ECRH to this baseline shot. The experimental temperature profiles of the discharges selected are shown for reference in grey. The temperature profiles are colour-coded by their on-axis values $T_{\mathrm{e}}(\rho=0)$.

\section{Impact of predisruption temperature on runaway}

The predisruption electron temperature is an important parameter for hot-tail runaway electron generation during the thermal quench, as discussed in $§ 4.2$. With increasing temperature, an exponentially increased hot-tail seed is expected to be generated. Simultaneously, increased impurity ionization is expected to occur under these conditions, potentially countering the increase of the hot-tail seed through enhanced friction. This behaviour is analysed computationally in this section by varying the preinjection, on-axis electron temperature in the range $T_{\mathrm{e}}(\rho=0) \in[4,20 \mathrm{keV}]$ in simulations of AUG \#33108.

\subsection{Set-up of electron temperature profiles}

In AUG runaway electron experiments, on-axis ECRH is applied in the last $0.1 \mathrm{~s}$ prior to impurity injection to achieve high electron temperatures in the vicinity of the magnetic axis. For AUG discharges similar to AUG \#33108, the electron temperature profiles obtained through Gaussian process regression of measurements by ECE and TS thus exhibit a peaked central temperature profile of varying magnitude (see figure $5 a$ ). For locations around midradius and beyond, the local temperature and the on-axis temperature are, however, not clearly correlated. This observation motivates the approach of constructing different experimentally relevant electron temperature profiles for this investigation based on the temperature profile of AUG \#33108 under the assumption of applying varying amounts of on-axis ECRH.

In contrast to using experimental temperature profiles of discharges with a desired preinjection on-axis temperature, this approach ensures applying temperature profiles consistent with each other throughout the temperature range considered, thus removing the impact peculiarities of the individual temperature profiles might have on the simulation results. Furthermore, this approach allows investigation of cases not covered (yet) experimentally, particularly at temperatures beyond $10 \mathrm{keV}$, while still ensuring experimental relevance. 
(a)

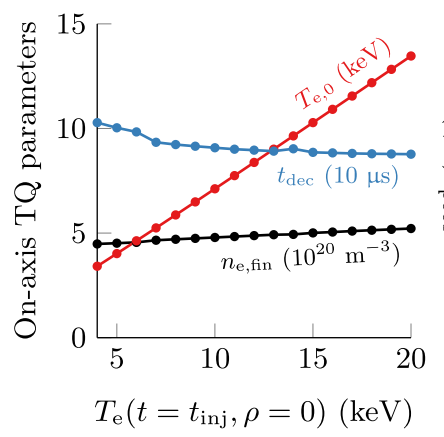

(b)

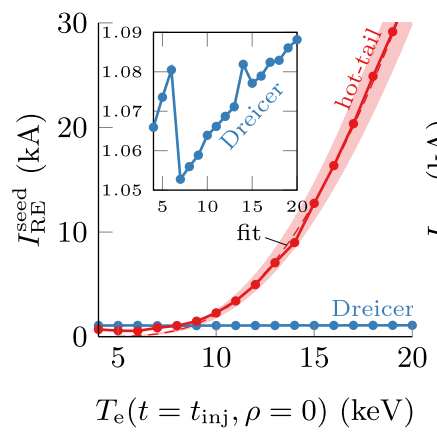

(c)

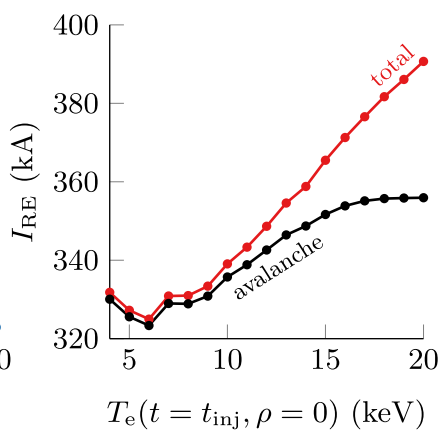

FIGURE 6. Simulations of AUG \#33108 with increasing preinjection on-axis electron temperature $T_{\mathrm{e}}\left(t=t_{\mathrm{inj}}, \rho=0\right)$, showing $(a)$ on-axis thermal quench parameters, being the postquench electron density (black), the electron temperature at the onset of the thermal quench (red) and the temperature decay time scale (blue). The runaway current obtained at the end of the disruption is shown for $(b)$ the seed runaway population $I_{\mathrm{RE}}^{\text {sed }}$, generated by the hot-tail mechanism (red) and by the Dreicer mechanism (blue), as well as for (c) the avalanche generated runaway current (black) and the total runaway current (red). The hot-tail current in panel $(b)$ is approximated by a function $I_{\mathrm{hot}}^{\mathrm{fit}}=a_{0} \exp \left(-a_{1} / T_{\mathrm{e}, 0}\right)$ (dashed red) of (5.2), with fitting parameters $a_{0}$ and $a_{1}$.

To construct the temperatures profiles used, the experimental temperature profile of AUG \#33108 is separated into a contribution $T_{\Omega}(\rho)$ due to Ohmic heating and into a contribution $T_{\mathrm{ECRH}}(\rho)$ due to ECRH (see figure $5 a$ ). Given the localized application, the ECRH contribution is non-vanishing only inside $\rho=0.35$. Profiles with an arbitrary electron temperature $T_{\mathrm{ax}}$ at the magnetic axis are thus obtained by scaling the ECRH contribution, according to

$$
T_{\mathrm{e}}(\rho)=\frac{T_{\mathrm{ax}}-T_{\Omega}(0)}{T_{\mathrm{ECRH}}(0)} T_{\mathrm{ECRH}}(\rho)+T_{\Omega}(\rho) .
$$

The temperature profiles constructed are consequently not modified beyond $\rho=0.35$. The profiles used throughout this scan in the range $T_{\mathrm{e}}(\rho=0) \in[4,20 \mathrm{keV}]$ are shown in figure $5(b)$. Compared to the temperature profiles of the discharges selected (illustrated in the same figure), the experimentally observed peaked temperature profiles are well described by the approach chosen. Therefore, the scan presented in the following describes experimentally relevant cases.

\subsection{Impact on the runaway seed}

Increasing the preinjection on-axis electron temperature $T_{\mathrm{e}}\left(t_{\mathrm{inj}}, 0\right)$ in simulations of AUG \#33108 from $4 \mathrm{keV}$ up to $20 \mathrm{keV}$, the hot-tail current generated is observed to grow exponentially from a minimum value of $0.6 \mathrm{kA}$ up to $33.7 \mathrm{kA}$ (see figure $6 b$ ). For the smallest choices of $T_{\mathrm{e}}\left(t_{\mathrm{inj}}, 0\right)$, runaway occurs predominantly around $\rho \sim 0.7$, shifting towards $\rho \sim 0.1$ with increasing $T_{\mathrm{e}}\left(t_{\mathrm{inj}}, 0\right)$ (see figure $7 a$ ). Here, the minimum hot-tail current is observed for $T_{\mathrm{e}}\left(t_{\mathrm{inj}}, 0\right)=6 \mathrm{keV}$. Significant hot-tail runaway eventually occurs for preinjection on-axis temperatures beyond $10 \mathrm{keV}$ (such that the hot-tail current constitutes more than $1 \%$ of the postdisruption runaway current), generating in all cases considered a seed current density noticeably smaller than the local predisruption Ohmic current density $j_{\Omega}$ (see figure 7). In the region of parameter space with $T_{\mathrm{e}}\left(t_{\mathrm{inj}}, 0\right)>10 \mathrm{keV}$, 


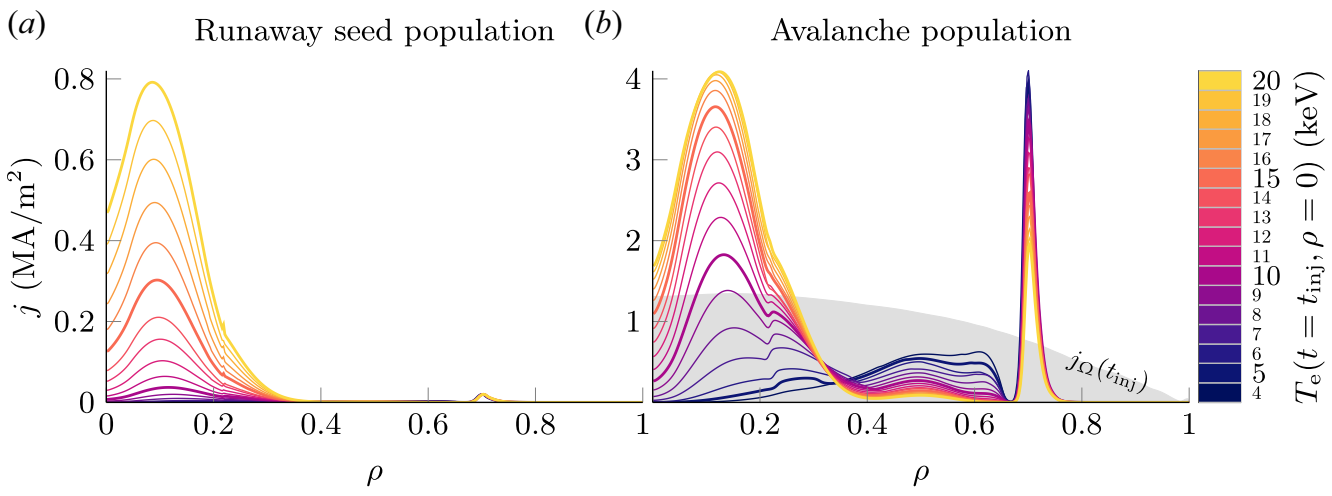

FIGURE 7. Radial profiles of (a) the runaway electron seed current densities $j_{\text {seed }}$ and $(b)$ the postdisruption runaway electron current densities $j_{\mathrm{av}}$ generated by the avalanche mechanism in simulations of AUG \#33108 with varying preinjection on-axis electron temperatures $T_{\mathrm{e}}(t=$ $t_{\text {inj }}, \rho=0$ ) ranging from 4 to $20 \mathrm{keV}$. For reference, the Ohmic current density $j_{\Omega}$ at the start of MGI is shown (grey) in panel $(b)$.

the hot-tail current obtained is well approximated by a function based on the simplified estimate of the hot-tail population (see (4.2)),

$$
I_{\text {hot }}^{\mathrm{fit}}\left(T_{\mathrm{e}, 0}(\rho=0)\right)=(914 \pm 58) \exp \left(-4\left\{\frac{\tilde{v} \ln \Lambda\left(t_{0}\right)\left\langle n_{\mathrm{e}, \mathrm{fin}} t_{\mathrm{dec}}\right\rangle}{T_{\mathrm{e}, 0}(\rho=0)^{3 / 2}}\right\}^{2 / 3}\right) \mathrm{kA},
$$

using the on-axis temperature $T_{\mathrm{e}, 0}(\rho=0)$ at the onset of the thermal quench as dependent variable. This estimate suggests an effective spatial average of the postquench electron density $n_{\mathrm{e}, \mathrm{fin}}$ and decay time scale $t_{\mathrm{dec}}$ of $\left\langle n_{\mathrm{e}, \mathrm{fin}} t_{\mathrm{dec}}\right\rangle=(1.66 \pm 0.04) \times 10^{19} \mathrm{~m}^{-3} \mathrm{~ms}$. Consequently, the hot-tail runaway current grows with increasing preinjection temperature as anticipated following the above argument. Yet, the free electron density due to impurity ionization does not increase at a similar rate (see figure $6(a)$ for the on-axis values of thermal quench parameters), due to the increasing ionization potential of higher impurity ion charge states. At the same time, the decay time scale decreases slightly for larger temperatures, thus partially compensating the increase of the electron density. As a result, hot-tail runaway strongly increases in hotter plasmas. It should be noted that the opposite effect was observed by Aleynikov \& Breizman (2017), where, different to the study presented in this manuscript, scenarios of instantaneous impurity deposition were investigated under the assumption of a steady-state impurity charge state distribution.

The impact of a variation of the preinjection on-axis electron temperature on electron runaway due to the Dreicer mechanism is, however, negligible (see figure $6 b$ ), varying only by approximately $3 \%$ throughout the temperature range considered. As discussed in $\$ 4.2$, Dreicer generation occurs as a result of the contracting Ohmic current predominantly in the vicinity of the $q=2$ surface, i.e. where the preinjection temperature profile is considered unaffected by a variation of on-axis ECRH. Consequently, the Dreicer generated runaway current is approximately constant in the scenario considered.

\subsection{Runaway electron multiplication}

Throughout the temperature range considered, the runaway current generated due to the avalanche mechanism increases (see figure $6 c$ ), but not in proportion to the strong increase of the hot-tail seed population. For preinjection on-axis temperatures below $10 \mathrm{keV}$, 
(a)

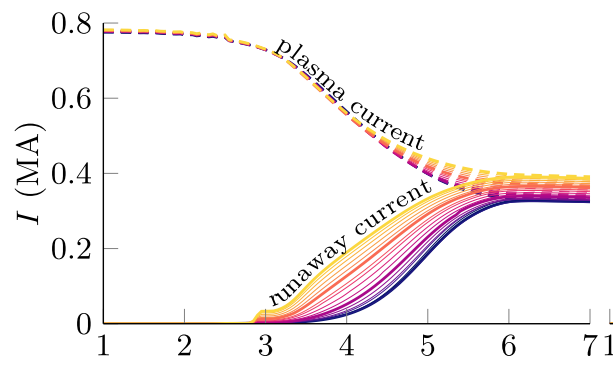

(c)

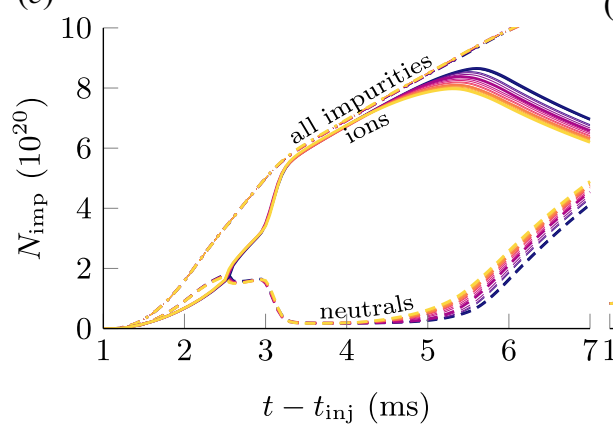

(b)

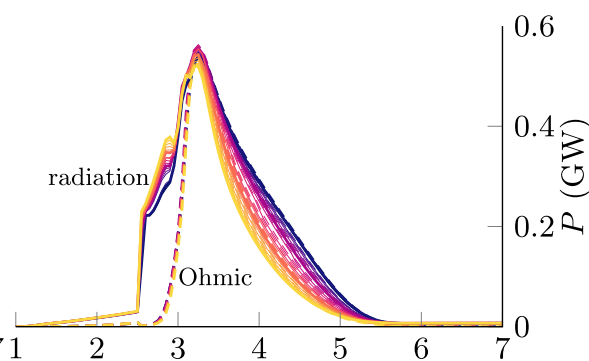

(d)

FIGURE 8. Temporal evolution of plasma parameters throughout the disruption in simulations of varying preinjection on-axis electron temperature $T_{\mathrm{e}}\left(t_{\mathrm{inj}}, 0\right)$, being $(a)$ the current $I$ of runaway electrons (solid) and of the plasma (dashed), (b) the power $P$ of Ohmic heating (dashed) and radiation due to both line radiation and Bremsstrahlung (solid), (c) impurity content $N_{\mathrm{imp}}$ of ionized impurities (solid), neutral impurities (dashed) and all impurities (dash-dotted), (d) volume-averaged effective charge (solid) and maximum effective charge (dashed).

the small variation of the runaway seed population results in an approximately constant avalanche current of around $330 \mathrm{kA}$. With the significant increase of the hot-tail population for larger temperatures, the avalanche generated current grows as well with temperature, yet noticeably only by a similar amount. In the range between 9 and $14 \mathrm{keV}$, the avalanche multiplication factor of the additional hot-tail population amounts to only between 2 and 3 , even approaching a factor of 1 for $T_{\mathrm{e}, 0} \rightarrow 20 \mathrm{keV}$. Consequently for temperatures above $17 \mathrm{keV}$, the avalanche generated current reaches a constant value of $356 \mathrm{kA}$.

The radial distribution of the avalanche current density changes throughout the range of preinjection temperatures (see figure $7 b$ ) as a result of the increasing hot-tail seed population close to the magnetic axis. Occurring predominantly in the vicinity of the $q=2$ surface and around midradius for lower temperatures, avalanche generation shifts towards the magnetic axis to around $\rho=0.12$. Given the large hot-tail seed in this region, significant avalanching starts earlier into the current quench, thus accelerating the decay of the residual Ohmic current.

The total runaway electron current obtained at the end of the disruption increases roughly linearly for $T_{\mathrm{e}, 0}>9 \mathrm{keV}$ (see figure $6 c$ ) due to the significantly growing hot-tail population. For the largest temperatures considered, the hot-tail seed constitutes almost $9 \%$ of the postdisruption runaway current. Consequently, the relative impact of avalanche multiplication decreases significantly with increasing temperature, as non-negligible amounts of the finite poloidal magnetic flux available for conversion to runaways (Boozer 2019) are consumed by a growing population of hot-tail runaways. 
Consequently, avalanche multiplication in future devices such as ITER may be less than predicted in previous studies (Hesslow et al. 2019a).

\subsection{Background plasma and impurity evolution}

The variation of the preinjection, on-axis temperature in the simulations discussed affects not only the spatio-temporal evolution of the runaway population, but also the evolution of the background plasma and of the impurities injected (see figure 8 for selected quantities). With higher initial temperature, impurity radiation (including line radiation and Bremsstrahlung) during the thermal quench is enhanced to dissipate the increased plasma thermal energy (see figure $8 b$ ). However, as the temperature profiles are effectively modified only in the region $\rho<0.3$ (see figure $5 b$ ), making up around $10 \%$ of the total plasma volume, the preinjection plasma stored thermal energy increases throughout the temperature range considered only by around $30 \%$. The net energy lost, being the difference between Ohmic heating and impurity radiation, until the end of the thermal quench is increased by the same amount.

During the thermal quench, radiative losses far exceed 200 MW throughout the temperature range considered. As such, conductive heat transport plays a marginal role in removing heat from the central plasma. The thermal quench is therefore induced by impurity radiation in the simulations performed. The duration of the thermal quench is similar in all cases, as inferred from the occurrence of a balance between impurity radiation and Ohmic heating. This is also manifested by the temperature decay time scale (see figure $6 a$ ), which decreases only slightly as larger preinjection temperatures are applied. The content of impurities inside the core plasma is identical during the thermal quench throughout the temperature range considered (see figure $8 c$ ). Consequently, larger densities of high impurity ionization stages are present at the end of the thermal quench in cases of high initial temperature (see figure $8 d$ ).

The seed population of hot-tail runaway electrons generated during the thermal quench increases in the central plasma as larger initial temperatures are applied (see $§ 5.3$ ). Consequently, noticeable avalanche generation starts earlier in the disruption in the high-temperature cases (see figure $8 a$ ). In the process, the residual Ohmic current is depleted quicker, providing reduced amounts of Ohmic heating to the cold postquench plasma (see figure 8b). As impurity radiation and Ohmic heating is balanced during the current quench, the impurities deposited in cases of hotter predisruption plasmas effectively recombine earlier into the current quench (see figures $8 c, d$ ). Nevertheless, the total length of the disruptions simulated is comparable throughout the temperature range considered, with the postdisruption runaway current being established at around $6 \mathrm{~ms}$ after the MGI valve trigger.

\subsection{Comparison with experimental observations}

For a comparison of the postdisruption runaway current for varying preinjection temperatures between the ASTRA-STRAHL simulations discussed above and AUG experiments, discharges similar to AUG experiment \#33108 are selected out of all runaway electron experiments performed in AUG. The selection is based on the preinjection plasma current, injection quantity, toroidal magnetic field and edge safety factor, according to the criteria listed in table 1 . The experimentally measured runaway current as a function of the preinjection temperature is shown for these discharges, as well as for all runaway electron experiments performed in AUG in figure 9. The preinjection, on-axis electron temperatures are determined applying GPR (see § 3.2) to central ECE measurements.

Experimental observations of the postdisruption runaway electron current as a function of the preinjection temperature show no clear correlation between both quantities 


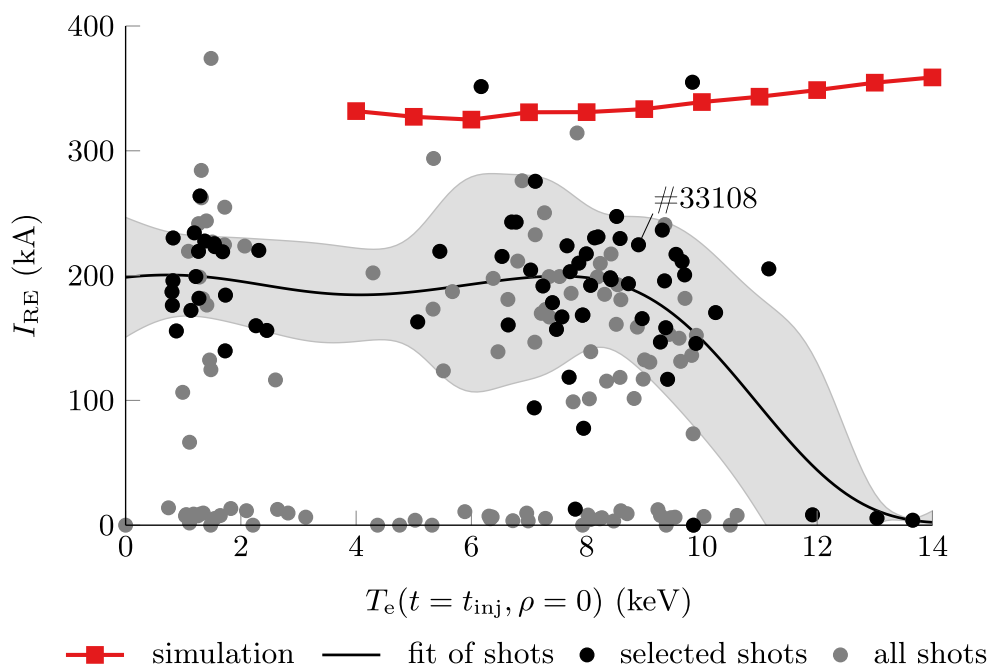

FIGURE 9. Postdisruption runaway electron current $I_{\mathrm{RE}}$ calculated in simulations of varying preinjection on-axis electron temperature $T_{\mathrm{e}, 0}$ (red squares) compared with the experimental dependence $I_{\mathrm{RE}}\left(T_{\mathrm{e}, 0}\right)$ of selected AUG shots similar to \#33108 (black circles) and of all runaway electron experiments performed in AUG (grey circles). Gaussian process regression of shots similar to AUG \#33108 shows the general trend observed experimentally (solid black), including uncertainties (filled grey).

(see figure 9). Runaway currents ranging from 150 to $250 \mathrm{kA}$ are generated regularly, but may also be as large as $350 \mathrm{kA}$ or may not be observed at all. Only for temperatures well above $10 \mathrm{keV}$, electron runaway does not occur. However, in this parameter region, only a small number of discharges has been performed. The experimentally observed relation between runaway current and postinjection temperature can be estimated applying GPR. Here, a runaway current of around $190 \mathrm{kA}$ independent of the temperature is on average expected for temperatures below $9 \mathrm{keV}$.

In the ASTRA-STRAHL simulations of AUG discharge \#33108 performed for varying preinjection temperatures, no strong temperature dependence of the postdisruption runaway current is observed for temperatures below $9 \mathrm{keV}$, similarly to the experimental estimate from Gaussian process regression. Yet, the calculated runaway current of around $330 \mathrm{kA}$ is noticeably larger than the experimental average. Still, this behaviour is expected, as the assumption regarding the average runaway electron velocity, $\left\langle v_{\mathrm{RE}}\right\rangle=$ $c$, may somewhat overestimate the runaway current, especially from contributions of runaways generated late into the current quench. However, a reduction of $\left\langle v_{\mathrm{RE}}\right\rangle / c$ will not proportionally reduce the postdisruption runaway current due to prolonged avalanche multiplication under these conditions (see Appendix B). As loss mechanisms for and radial transport of runaway electrons are also not considered in this work, the runaway current calculated is expected to be further overestimated. Thus, the simulations provide a pessimistic estimate of the runaway electron current. Under consideration of these effects, the relative contributions of individual generation mechanisms are expected to change only marginally, preserving the trends observed in the simulations. Importantly, neither in experiments, nor in simulations, a pronounced temperature dependence of the runaway current is observed for temperatures below $9 \mathrm{keV}$. 
For temperatures above $10 \mathrm{keV}$, simulations predict a steadily increasing postdisruption runaway current, contrary to experimental observations of a vanishing runaway current. As runaway generation in all simulations occurs predominantly due to the avalanche mechanism, the absence of a postdisruption runaway current in the experiment suggests that no seed population is present at the end of thermal quench. After all, significant avalanche multiplication of a runaway seed is expected during the current quench, given that the parallel electric field typically far exceeds the effective critical electric field under these conditions. As the amount of material injected is similar in all experiments selected, the impurity friction experienced by highly energetic electrons is assumed not to be increased. Conditions for avalanche multiplication are therefore also expected suitable for preinjection temperatures above $10 \mathrm{keV}$.

Assuming favourable conditions for avalanche multiplication, the absence of a runaway seed population is due to either insufficient generation of primary runaways or due to the loss of the entire seed during breakup of the magnetic surfaces. However, the generation models employed in this work predict the formation of a noticeable seed population. Generation due to the Dreicer mechanism is driven by the contracting Ohmic current density in the vicinity of the $q=2$ surface. Therefore, the modification of the central electron temperature profile affects electron runaway due to momentum-space diffusion in this particular region only insignificantly, even under consideration of radial broadening of the electron temperature profile during application of increasing amounts of ECRH. In the case of hot-tail runaway, an increase of the preinjection temperature strongly facilitates formation of a seed population, particularly under the assumption of radial broadening of the temperature profile. Thus, for preinjection temperatures beyond $10 \mathrm{keV}$, formation of a noticeable runaway seed is also expected to occur. Note that the exponential time scale $t_{\mathrm{dec}}$ and, more importantly, the variation thereof throughout the temperature range considered cannot be determined experimentally in AUG with the available diagnostics due to insufficient temporal resolution of the TS diagnostic and the ECE signal being in high-density cutoff.

The absence of a runaway current in AUG experiments with preinjection temperatures above $10 \mathrm{keV}$ thus suggests, following the above argument, that the runaway seed is lost entirely during breakup of magnetic surfaces, i.e. before avalanche multiplication significantly increases the runaway population. Within ASTRA-STRAHL, this hypothesis cannot be tested as magnetic field line stochasticity and the associated runaway loss cannot be modelled self-consistently in this framework. Instead, non-linear MHD codes could be applied to investigate the existence of a transition in field line stochasticity in AUG disruptions when increasing the preinjection temperature to above $10 \mathrm{keV}$. Similarly, the signals of magnetic diagnostics in AUG disruption experiments of varying core temperature should be analysed in future work regarding changes in MHD activity.

It should be noted that, analysing AUG runaway experiments with preinjection temperatures above $10 \mathrm{keV}$, the low number of available discharges is not sufficient to rule out the existence of a postdisruption runaway current in this temperature region. After all, for the discharges selected, the absence of a postdisruption runaway current is also occasionally observed for temperatures below $10 \mathrm{keV}$. Therefore, further experiments with strongly increased temperatures are required to confirm or disprove the general absence of a postdisruption runaway current under these conditions.

The question of electron runaway at temperatures well above $10 \mathrm{keV}$ is especially relevant for future fusion devices, such as ITER. If the runaway seed is indeed lost completely during high-temperature disruptions, the risk of producing a large runaway electron current would be greatly reduced. In the opposite case, the runaway seed 
generated by the hot-tail mechanism is expected to contribute significantly to the overall plasma current. If, simultaneously, seed losses were to be increased (through external manipulation), poloidal flux could be removed effectively and thus avalanche multiplication hindered.

\section{Conclusion}

In this work, runaway electron generation in ASDEX Upgrade MGI experiments was investigated by means of 1.5-D transport simulations performed with the coupled codes ASTRA-STRAHL. The suitability of this approach for the study of electron runaway in ASDEX Upgrade has recently been demonstrated by Linder et al. (2020). For this study, the toolkit chosen has been extended by a model from Smith \& Verwichte (2008) describing the hot-tail population during the thermal collapse of the plasma.

Applied in simulations of argon injection in ASDEX Upgrade discharge \#33108, primary runaway generation mechanisms are calculated to both produce only a small seed population of comparable magnitude, being in total around $3 \mathrm{kA}$ of fast electrons. Whereas electron runaway due to the Dreicer mechanism occurs as a result of the inward contracting Ohmic current predominantly in the vicinity of the $q=2$ surface at $\rho \sim 0.7$ prior to thermal collapse, hot-tail runaway is encountered primarily in the central plasma during the thermal quench as the postcollapse hot-tail population is exponentially sensitive to the predisruption temperature, $n_{\text {hot }}\left(t_{\text {fin }}\right) \propto \exp \left(-1 / T_{\mathrm{e}, 0}\right)$. At the end of the disruption, a runaway current of $331 \mathrm{kA}$ is obtained in the simulations, the vast majority generated by the avalanche mechanism. A similar impact of runaway generation mechanisms has also been observed in kinetic simulations with the full- $f$ solver CODE (Insulander Björk et al. 2020). In the simulations presented in this work, similar postdisruption runaway electron currents are generated when neglecting one of the primary generation mechanisms. Thus, avalanche multiplication plays a significant role for the formation of a postdisruption runaway current in ASDEX Upgrade.

Investigating the impact of varying the central electron temperature prior to argon injection in these scenarios, the postdisruption runaway current is approximately constant for on-axis temperatures below $9 \mathrm{keV}$ in both simulations and experiment, generating a runaway current of around 330 and $190 \mathrm{kA}$, respectively. Differences are assumed to be due to the absence of runaway loss mechanisms and an overestimation of the average runaway electron velocity, $\left\langle v_{\mathrm{RE}}\right\rangle=c$. For larger temperatures up to $20 \mathrm{keV}$, simulations predict a strongly increased hot-tail population and consequently an increase of the postdisruption runaway current. Contradictorily, in the few ASDEX Upgrade discharges available in this parameter region, no postdisruption runaway current is detected. As the runaway electron models predict strong primary and secondary generation under these conditions, the absence of a postdisruption current in the experiment is considered to be caused by the loss of the entire seed population. Here, non-linear MHD codes could be applied to investigate if field line stochasticity drastically enhances seed losses. Furthermore, analysis of MHD activity inferred from measurements by magnetic diagnostics should be performed in future studies. Finally, further runaway electron experiments in ASDEX Upgrade are required to confirm or disprove the experimental trend observed.

In the simulations performed, the hot-tail mechanism provides only a small seed population of runaway electrons. Yet, the model by Smith \& Verwichte (2008) employed is known to underestimate the hot-tail density (Stahl et al. 2016). Under application of more elaborate (kinetic) models, hot-tail runaway is thus suspected to be significantly increased. Simultaneously, in scenarios such as ASDEX Upgrade discharge \#33108, the postdisruption runaway current is not expected to be drastically increased. However in 
hotter predisruption plasmas, a more realistic description of hot-tail runaway could provide a substantial seed population. Simultaneously, magnetic perturbations could significantly reduce avalanche multiplication (Svensson et al. 2021). As this temperature range is relevant for future fusion devices, further investigation of primary runaway under these conditions is required. Here, reduced kinetic models, as, for example, being developed by Svenningsson (2020), could be employed in combination with radial runaway transport coefficients, e.g. by Särkimäki et al. (2020), to consider runaway losses.

\section{Supplementary movie}

Supplementary movie is available at https://doi.org/10.1017/S0022377821000416.

\section{Acknowledgements}

The authors would like to thank J. Hobirk for developing the high-temperature scenarios and M. Hoelzl for clarifying thoughts on MHD mode activity during the thermal quench.

\section{Editor Tünde Fülöp thanks the referees for their advice in evaluating this article.}

\section{Funding}

This work was supported by the EUROfusion - Theory and Advanced Simulation Coordination (E-TASC). This work has been carried out within the framework of the EUROfusion Consortium and has received funding from the Euratom research and training programme 2014-2018 and 2019-2020 under grant agreement No 633053. The views and opinions expressed herein do not necessarily reflect those of the European Commission.

\section{Declaration of interests}

The authors report no conflict of interest.

\section{Appendix A. Approximation of the hot-tail density}

The temporal evolution of the hot-tail runaway electron density $n_{\text {hot }}(t)$ throughout the thermal quench can be calculated with the model by Smith \& Verwichte (2008). Yet to assess the density only at the end of the thermal quench at $t_{\text {fin }}$, evaluation of the model from onset of the quench at $t_{0}$ until $t_{\text {fin }}$ is still required. Alternatively, a simple estimate can be obtained considering only the dominating contributions to the hot-tail density.

Considering the full expression (see (2.3))

$$
n_{\mathrm{hot}}(t)=\frac{4 n_{\mathrm{e}, 0}}{\sqrt{\pi} v_{\mathrm{th}, 0}^{3}} \int_{v_{\mathrm{c}}(t)}^{\infty}\left(v^{2}-v_{\mathrm{c}}(t)^{2}\right) \exp \left(-\left[\frac{v^{3}}{v_{\mathrm{th}, 0}^{3}}+3 \tau(t)\right]^{2 / 3}\right) \mathrm{d} v,
$$

the velocity distribution function is evaluated beyond the critical velocity for runaway $v_{\mathrm{c}}^{2}=e^{3} n_{\mathrm{e}} \ln \Lambda / 4 \pi \varepsilon_{0}^{2} m_{\mathrm{e}} E_{\|}$. Throughout the process of thermal collapse, an initially large $v_{\mathrm{c}}\left(t_{0}\right) \gg v_{\mathrm{th}, 0}$ will eventually approach $v_{\mathrm{th}, 0}$, i.e. $v_{\mathrm{c}}\left(t_{\mathrm{fin}}\right) \rightarrow v_{\mathrm{th}, 0}$, as the local electric field $E_{\|}$strongly increases. Simultaneously, the parameter $\tau(t)$ grows throughout the quench according to $\tau(t)=\left(t-t_{\mathrm{dec}}\right) v_{0} n_{\mathrm{e}, \text { fin }} / n_{\mathrm{e}, 0}$. Consequently, the exponent of the exponential function of (A 1) starts typically far from unity, i.e. $v_{\mathrm{c}}^{3} / v_{\mathrm{th}, 0}^{3}+3 \tau \gg 1$. As the exponential function decreases rapidly for $v>v_{\mathrm{c}}$, electrons with $v \gtrsim v_{\mathrm{c}}$ contribute dominantly to the velocity-space integral. Under these assumptions, the argument of the exponential function in (A 1) can be approximated as

$$
-\left[\frac{v^{3}}{v_{\mathrm{th}, 0}^{3}}+3 \tau(t)\right]^{2 / 3} \approx-\left(\frac{v}{v_{\mathrm{c}}(t)}\right)^{2}\left[\left(\frac{v_{\mathrm{c}}(t)}{v_{\mathrm{th}, 0}}\right)^{3}+3 \tau(t)\right]^{2 / 3} .
$$


The solution of the velocity-space integral is thus readily obtained as

$$
n_{\mathrm{hot}}(t)=\frac{2 n_{\mathrm{e}, 0}}{\sqrt{\pi}} \frac{\left(\frac{v_{\mathrm{c}}(t)}{v_{\mathrm{th}, 0}}\right)^{3}}{\left[\left(\frac{v_{\mathrm{c}}(t)}{v_{\mathrm{th}, 0}}\right)^{3}+3 \tau(t)\right]^{2 / 3}} \exp \left(-\left[\left(\frac{v_{\mathrm{c}}(t)}{v_{\mathrm{th}, 0}}\right)^{3}+3 \tau(t)\right]^{2 / 3}\right) .
$$

A similar approximation is derived in Smith \& Verwichte (2008), where the numerator of the pre-exponential fraction is amended by $+3 \tau(t)$. However, the resulting expression overestimates the hot-tail population, compared with evaluation of (A 1).

To obtain the postquench hot-tail population, the above expression has to be evaluated at time $t^{\star}$, when the exponent of the exponential function reaches its maximum value. This in turn requires evaluation of the temporal evolution of the critical velocity $v_{\mathrm{c}}(t)$. Considering an exponential decay of the electron temperature in the limit $T_{\mathrm{e}, \text { fin }} \ll T_{\mathrm{e}, 0}$, i.e. $T_{\mathrm{e}}(t)=T_{\mathrm{e}, \text { fin }}+\left(T_{\mathrm{e}, 0}-T_{\mathrm{e}, \text { fin }}\right) \mathrm{e}^{-t / t_{\mathrm{dec}}} \approx T_{\mathrm{e}, 0} \mathrm{e}^{-t / t_{\mathrm{dec}}}$, the evolution of the electric field required for $v_{\mathrm{c}}(t)$ is obtained as

$$
E_{\|}(t)=\frac{j_{\Omega, 0}}{\sigma(t)}=\frac{e^{2} \sqrt{m_{\mathrm{e}}} \ln \Lambda}{8 \sqrt{2} \pi \varepsilon_{0}^{2} T_{\mathrm{e}}(t)^{3 / 2}} j_{\Omega, 0}=E_{\|, 0} \exp \left(\frac{3}{2} \frac{t}{t_{\mathrm{dec}}}\right),
$$

with $\sigma$ being the plasma conductivity. Note, that the Ohmic current density $j_{\Omega}(t)$ is in good approximation constant throughout the part of the thermal collapse relevant for hot-tail runaway. Alternatively, the evolution of the electric field can be evaluated through $(\mathrm{d} / \mathrm{d} t)\left\{\sigma(t) E_{\|}(t)\right\}=-2 R E_{\|}(t) / L a^{2}$ (Hesslow et al. 2018b), with major radius $R$, minor radius $a$ and inductance $L$. Using typical AUG parameters, deviations with respect to expression (A 4 ) become important only for $t / t_{\mathrm{dec}} \gtrsim 5$, i.e. when the hot-tail population is already established. Thus, the ratio of velocities can be written as

$$
\frac{v_{\mathrm{c}}(t)}{v_{\mathrm{th}, 0}}=\sqrt{\frac{e n_{\mathrm{e}, 0}}{j_{\Omega, 0}} \sqrt{\frac{2 T_{\mathrm{e}, 0}}{m_{\mathrm{e}}}}} \exp \left(-\frac{3}{4} \frac{t}{t_{\mathrm{dec}}}\right)=\frac{v_{\mathrm{c}, 0}}{v_{\mathrm{th}, 0}} \exp \left(-\frac{3}{4} \frac{t}{t_{\mathrm{dec}}}\right)
$$

and thus the exponent of the exponential function obtains its maximum value at time (see also Smith \& Verwichte 2008)

$$
t^{\star}=\frac{4}{9} t_{\mathrm{dec}}\left\{3 \log \left(\frac{v_{\mathrm{c}, 0}}{v_{\mathrm{th}, 0}}\right)-\log \left(\frac{4}{3} v_{0} \frac{n_{\mathrm{e}, \mathrm{fin}}}{n_{\mathrm{e}, 0}} t_{\mathrm{dec}}\right)\right\} .
$$

Applied in (A 3), the hot-tail population at the end of the thermal quench can be estimated as

$$
n_{\mathrm{hot}}\left(t_{\mathrm{fin}}\right) \approx \frac{2 n_{\mathrm{e}, 0}}{\sqrt{\pi}} \frac{\mathcal{F}^{1 / 3}}{\mathcal{G}^{2 / 3}} \exp \left(-(\mathcal{F} \mathcal{G})^{2 / 3}\right)
$$

where

$$
\mathcal{F}=\frac{4}{3} v_{0} \frac{n_{\mathrm{e}, \mathrm{fin}}}{n_{\mathrm{e}, 0}} t_{\mathrm{dec}}, \quad \mathcal{G}=3 \log \left(\frac{v_{\mathrm{c}, 0}}{v_{\mathrm{th}, 0}}\right)-\log \mathcal{F}-\frac{5}{4} .
$$

The radial variation of (A 7) is primarily determined by the contribution of $\mathcal{F}$ in the exponent of the exponential function. To emphasize this dependence, further simplifications can be made by applying values for the disruption parameters as typically 


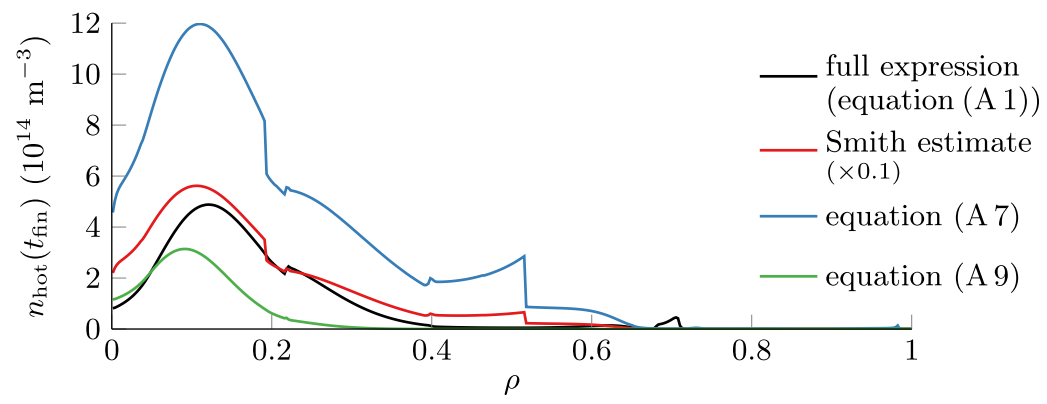

FIGURE 10. Hot-tail population of AUG \#33108 calculated in simulations of ASTRA-STRAHL evaluating the full expression of (A 1) (black), see $\S 4$, compared with analytical estimates from (23) of Smith \& Verwichte (2008) (red), (A 7) (blue) and (A 9) (green). Note that the estimate using the approximation by Smith \& Verwichte (2008) is scaled by a factor of $\times 0.1$.

occurring in AUG disruption experiments, yielding a pre-exponential factor of $\mathcal{F} / \mathcal{G}^{2} \approx 1$ and inside the exponent $(4 \mathcal{G} / 3)^{2 / 3} \approx 4$. Writing the collision frequency as $v_{0} n_{\mathrm{e}, \text { fin }} / n_{\mathrm{e}, 0} \equiv$ $\tilde{v} \ln \Lambda\left(t_{0}\right) n_{\mathrm{e}, \text { fin }} / T_{\mathrm{e}, 0}^{3 / 2}$, the hot-tail runaway density can thus be expressed as

$$
n_{\text {hot }}^{\text {simple }}\left(t_{\text {fin }}\right)=\frac{2 n_{\mathrm{e}, 0}}{\sqrt{\pi}} \exp \left(-4\left\{\tilde{\nu} \ln \Lambda\left(t_{0}\right) \frac{n_{\mathrm{e}, \mathrm{fin}} t_{\mathrm{dec}}}{T_{\mathrm{e}, 0}^{3 / 2}}\right\}^{2 / 3}\right) .
$$

Albeit being a simple estimate for the hot-tail population, key dependencies on thermal quench parameters are readily clear evaluating this expression. Estimates of the hot-tail population for the simulation of AUG discharge \#33108 presented in $\S 4$ are shown in figure 10 for the different analytical expressions introduced. The simple estimate of (A 9) agrees rather well with the hot-tail population obtained through evaluation of the full expression (A 1), thus illustrating the suitability of (A 9) to assess the dependence of the hot-tail population on parameters of the thermal quench.

\section{Appendix B. Average runaway electron velocity}

In the simulations presented, the runaway electron current density $j_{\mathrm{RE}}$ is calculated from the number density $n_{\mathrm{RE}}$ under the assumption that runaway electrons travel with the speed of light, i.e. the average runaway electron velocity $\left\langle v_{\mathrm{RE}}\right\rangle=c$. For large kinetic energies $E_{\text {kin }}>6.1 m_{\mathrm{e}} c^{2}=3.1 \mathrm{MeV}$, this gives a less than $1 \%$ error. However, the validity of this assumption is often questioned. Therefore, it is demonstrated in this section that a reasonable choice of $\left\langle v_{\mathrm{RE}}\right\rangle \sim c$ has only a minor impact on the amount of postdisruption runaway current generated.

In simulations of AUG \#33108 of varying average runaway electron velocity, the postdisruption runaway electron current obtained is rather insensitive to a moderate modification of $\left\langle v_{\mathrm{RE}}\right\rangle$, as shown in figure 11. Note, that these simulations presented were performed with decreased temporal resolution for illustrative purposes. Assuming an average velocity of $50 \% \mathrm{c}$, the postdisruption runaway current is reduced by $15 \%$. Only for $\left\langle v_{\mathrm{RE}}\right\rangle \lesssim 20 \% c$, the runaway current calculated falls off quickly. Importantly, the associated kinetic energy of the runaway electrons is well below $m_{\mathrm{e}} c^{2}$ for both choices of $\left\langle v_{\mathrm{RE}}\right\rangle / c$ discussed, approaching even the predisruption thermal electron energy. However as the bulk of the runaway electron population is expected to reach kinetic energies above the rest mass energy, corresponding to $\left\langle v_{\mathrm{RE}}\right\rangle>87 \% c$, the postdisruption runaway current is not significantly affected by a variation of $\left\langle v_{\mathrm{RE}}\right\rangle$ within these bounds. 


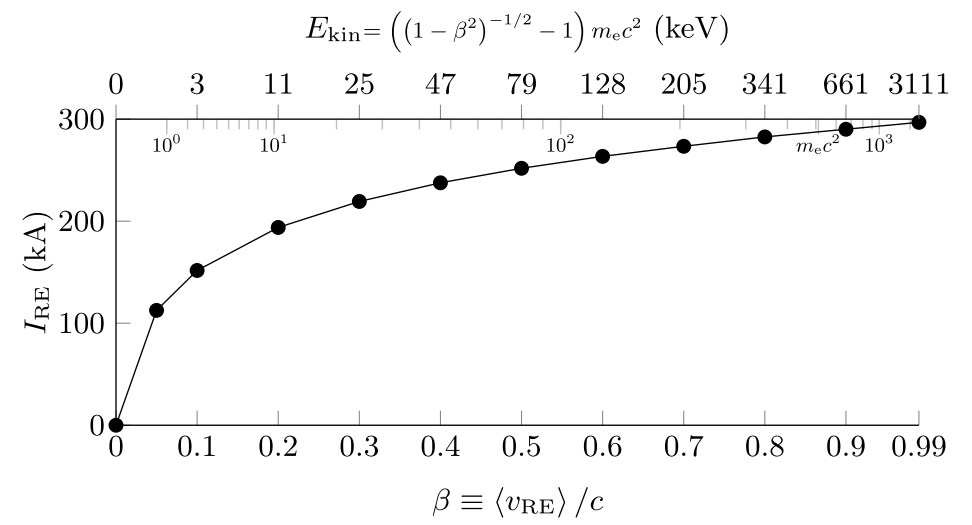

FIGURE 11. Postdisruption runaway electron current $I_{\text {RE }}$ in simulations of AUG \#33108 applying a varying average runaway electron velocity $\left\langle v_{\mathrm{RE}}\right\rangle$. For reference, the corresponding kinetic electron energy $E_{\text {kin }}$ is given. Note, that these simulations were carried out with decreased temporal resolution for illustrative purposes.

A variation of the average runaway electron velocity can also be considered as a variation of the strength of primary runaway electron generation under the assumption $\left\langle v_{\mathrm{RE}}\right\rangle=c$. Writing $\beta \equiv\left\langle v_{\mathrm{RE}}\right\rangle / c$, the macroscopic transport equation (2.1) for the primary runaway electron current density can be expressed as (neglecting radial transport)

$$
\frac{\partial j_{\mathrm{seed}}}{\partial t}=e\left\langle v_{\mathrm{RE}}\right\rangle S_{\mathrm{seed}}=e(c \beta) S_{\mathrm{seed}}=e c\left(\beta S_{\mathrm{seed}}\right)
$$

hence describing either a variation of $\left\langle v_{\mathrm{RE}}\right\rangle$ or of $S_{\text {seed }}$. Simultaneously, avalanche multiplication is described by

$$
\frac{\partial j_{\mathrm{av}}}{\partial t}=e\left\langle v_{\mathrm{RE}}\right\rangle n_{\mathrm{RE}} \tilde{S}_{\mathrm{av}}=\left(j_{\mathrm{av}}+j_{\mathrm{seed}}\right) \tilde{S}_{\mathrm{av}},
$$

thus not explicitly considering assumptions regarding the average runaway electron velocity. A variation of $\left\langle v_{\mathrm{RE}}\right\rangle$ does therefore directly affect only the primary population. Hence, varying $\beta$ within the range $[0,1]$, the source strength $S_{\text {seed }}$ can be considered scaled by this factor instead of $\left\langle v_{\mathrm{RE}}\right\rangle$. Under these conditions of decreased primary generation, however, avalanche multiplication is not affected proportionally, as discussed in $\S 4.3$.

\section{REFERENCES}

Aleynikov, P. \& BReizman, B. N. 2017 Generation of runaway electrons during the thermal quench in tokamaks. Nucl. Fusion 57, 046009. https://doi.org/10.1088/1741-4326/aa5895.

Bandaru, V., Hoelzl, M., Artola, F. J., Papp, G. \& Huijsmans, G. T. A. 2019 Simulating the nonlinear interaction of relativistic electrons and tokamak plasma instabilities: Implementation and validation of a fluid model. Phys. Rev. E 99, 063317. https://doi.org/10.1103/PhysRevE.99.063317.

Boozer, A. H. 2019 Magnetic surface loss and electron runaway. Plasma Phys. Control. Fusion 61, 024002. https://doi.org/10.1088/1361-6587/aaf293.

Breizman, B. N., Aleynikov, P., Hollmann, E. M. \& Lehnen, M. 2019 Physics of runaway electrons in tokamaks. Nucl. Fusion 59, 083001. https://doi.org/10.1088/1741-4326/ab1822.

Chilenski, M. A., Greenwald, M., Marzouk, Y., Howard, N. T., White, A. E., Rice, J. E. \& WALK, J. R. 2015 Improved profile fitting and quantification of uncertainty in experimental measurements of impurity transport coefficients using Gaussian process regression. Nucl. Fusion 55, 023012. https://doi.org/10.1088/0029-5515/55/2/023012. 
Chiu, S. C., Rosenbluth, M. N., Harvey, R. W. \& Chan, V. S. 1998 Fokker-Planck simulations mylb of knock-on electron runaway avalanche and bursts in tokamaks. Nucl. Fusion 38, 1711. https://doi.org/10.1088/0029-5515/38/11/309.

Coda, S., Agostini, M., Albanese, R., Alberti, S., Alessi, E., Allan, S., Allcock, J., Ambrosino, R., Anand, H., the EUROfusion MST1 team, et al. 2019 Physics research on the TCV tokamak facility: from conventional to alternative scenarios and beyond. Nucl. Fusion 59, 112023. https://doi.org/10.1088/1741-4326/ab25cb.

Commaux, N., Baylor, L. R., Jernigan, T. C., Hollmann, E. M., Parks, P. B., Humphreys, D. A., Wesley, J. C. \& YU, J. H. 2010 Demonstration of rapid shutdown using large shattered deuterium pellet injection in DIII-D. Nucl. Fusion 50, 112001. https://doi.org/10.1088/0029-5515/50/11/112001.

Connor, J. W. \& Hastie, R. J. 1975 Relativistic limitations of runaway electrons. Nucl. Fusion 15, 415. https://doi.org/10.1088/0029-5515/15/3/007.

Dux, R., Peeters, A. G., Gude, A., Kallenbach, A. \& Neu, R. \& the ASdeX Upgrade Team $1999 \mathrm{Z}$ dependence of the core impurity transport in ASDEX Upgrade $\mathrm{H}$ mode discharges. Nucl. Fusion 39, 1509. https://doi.org/10.1088/0029-5515/39/11/302.

Fable, E., Angioni, C., Ivanov, A. A., Lackner, K., Maj, O., Medvedev, S. Y., Pautasso, G., Pereverzev, G. V. \& Treutterer, W. \& the ASDeX Upgrade Team 2013 Dynamical coupling between magnetic equilibrium and transport in tokamak scenario modelling, with application to current ramps. Plasma Phys. Control. Fusion 55, 074007. https://doi.org/10.1088/0741-3335/55/7/074007.

Fable, E., Pautasso, G., Lehnen, M., Dux, R., Bernert, M. \& Mlynek, A. \& THE ASDEX UPGRADE TEAM 2016 Transport simulations of the pre-thermal-quench phase in ASDEX Upgrade massive gas injection experiments. Nucl. Fusion 56, 026012. https://doi.org/10.1088/0029-5515/56/2/026012.

FehÉr, T., SMith, H. M., FÜlÖP, T. \& GÁL, K. 2011 Simulation of runaway electron generation during plasma shutdown by impurity injection in ITER. Plasma Phys. Control. Fusion 53, 035014. https://doi.org/10.1088/0741-3335/53/3/035014.

Harvey, R. W., Chan, V. S., Chiu, S. C., Evans, T. E., Rosenbluth, M. N. \& Whyte, D. G. 2000 Runaway electron production in DIII-D killer pellet experiments, calculated with the CQL3D/KPRAD model. Phys. Plasmas 7, 4590. https://doi.org/10.1063/1.1312816.

Harvey, R. W., Petrov, Y. V., Kim, C. C., Forest, C. B., Lao, L. L. \& Parks, P. B. 2019 Time-dependent runaway electron simulations: Ampere-Faraday equations implemented in CQL3D. Nucl. Fusion 59, 106046. https://doi.org/10.1088/1741-4326/ab38cb.

Hesslow, L., Embréus, O., Hoppe, M., Dubois, T. C., Papp, G., Rahm, M. \& Fülöp, T. $2018 a$ Generalized collision operator for fast electrons interacting with partially ionized impurities. J. Plasma Phys. 84, 905840605. https://doi.org/10.1017/S0022377818001113.

Hesslow, L., Embréus, O., Vallhagen, O. \& FÜlöP, T. $2019 a$ Influence of massive material injection on avalanche runaway generation during tokamak disruptions. Nucl. Fusion 59, 084004. https://doi.org/10.1088/1741-4326/ab26c2.

Hesslow, L., Embréus, O., Wilkie, G. J., PAPP, G. \& FülÖP, T. $2018 b$ Effect of partially ionized impurities and radiation on the effective critical electric field for runaway generation. Plasma Phys. Control. Fusion 60, 074010. https://doi.org/10.1088/1361-6587/aac33e.

Hesslow, L., Unnerfelt, L., Vallhagen, O., Embréus, O., Hoppe, M., Papp, G. \& Fülöp, T. $2019 b$ Evaluation of the Dreicer runaway growth rate in the presence of high- $Z$ impurities using a neural network. J. Plasma Phys. 85, 475850601. https://doi.org/10.1017/S0022377819000874.

Ho, A., Citrin, J., Auriemma, F., Bourdelle, C., Casson, F. J., Kim, H.-T., Manas, P., SzePesi, G. \& Weisen, H. \& JET CONTRIbUtors 2019 Application of Gaussian process regression to plasma turbulent transport model validation via integrated modelling. Nucl. Fusion 59, 056007. https://doi.org/10.1088/1741-4326/ab065a.

Hoppe, M., Hesslow, L., Embreus, O., Unnerfelt, L., Papp, G., Pusztai, I., Fülöp, T., LeXell, O., Lunt, T., Macusova, E., et al. 2021 Spatiotemporal analysis of the runaway distribution function from synchrotron images in an ASDEX Upgrade disruption. J. Plasma Phys. 87, 855870102. https://doi.org/10.1017/S002237782000152X. 
Insulander Björk, K., Papp, G., Embreus, O., Hesslow, L., FülöP, T., Vallhagen, O., Lier, A., Pautasso, G., Bock, A., the ASDeX Upgrade Team, et al. 2020 Kinetic modelling of runaway electron generation in argon-induced disruptions in ASDEX Upgrade. J. Plasma Phys. 86, 855860401. https://doi.org/10.1017/S0022377820000793.

Lehnen, M., Aleynikova, K., Aleynikov, P. B., Campbell, D. J., Drewelow, P., Eidietis, N. W., Gasparyan, Y., Granetz, R. S., Gribov, Y., Hartmann, N., et al. 2015 Disruptions in ITER and strategies for their control and mitigation. J. Nucl. Mater. 463, 39. https://doi.org/10.1016/j.jnucmat.2014.10.075.

Linder, O., Fable, E., Jenko, F., Papp, G. \& Pautasso, G., the AsdeX Upgrade Team \& THE EUROFUSION MST1 TEAM 2020 Self-consistent modeling of runaway electron generation in massive gas injection scenarios in ASDEX Upgrade. Nucl. Fusion 60, 096031. https://doi.org/10.1088/1741-4326/ab9dcf.

Martín-Solís, J. R., Loarte, A., Hollmann, E. M., Esposito, B. \& Riccardo, V., FTU Team, DIII-D TEAM \& JET EFDA CONTRIBUTORS 2014 Inter-machine comparison of the termination phase and energy conversion in tokamak disruptions with runaway current plateau formation and implications for ITER. Nucl. Fusion 54, 083027. https://doi.org/10.1088/0029-5515/54/8/083027.

Matthews, G. F., Bazylev, B., Baron-Wiechec, A., Coenen, J., Heinola, K., Kiptily, V., MAier, H., ReUX, C., RicCARdo, V., Rimini, F., et al. 2016 Melt damage to the JET ITER-like Wall and divertor. Phys. Scr. T167, 014070. https://doi.org/10.1088/0031-8949/T167/1/014070.

Papp, G., FÜlöP, T., Fehér, T., De Vries, P. C., Riccardo, V., ReuX, C., Lehnen, M., Kiptily, V., Plyusnin, V. V., AlPer, B., et al. 2013 The effect of ITER-like wall on runaway electron generation in JET. Nucl. Fusion 53, 123017. https://doi.org/10.1088/0029-5515/53/12/123017.

Pautasso, G., Bernert, M., Dibon, M., Duval, B., Dux, R., Fable, E., Fuchs, J. C., Conway, G. D., Giannone, L., Gude, A., et al. 2017 Disruption mitigation by injection of small quantities of noble gas in ASDEX Upgrade. Plasma Phys. Control. Fusion 59, 014046. https://doi.org/10.1088/0741-3335/59/1/014046.

Pautasso, G., Dibon, M., Dunne, M., Dux, R., Fable, E., Lang, P., Linder, O., Mlynek, A., PAPP, G., BERnERT, M., et al. 2020 Generation and dissipation of runaway electrons in ASDEX Upgrade experiments. Nucl. Fusion 60, 086011. https://doi.org/10.1088/1741-4326/ab9563.

Paz-Soldan, C., Aleynikov, P., Hollmann, E. M., Lvovskiy, A., Bykov, I., Du, X., Eidietis, N. W. \& SHIRAKI, D. 2020 Runaway electron seed formation at reactor-relevant temperature. Nucl. Fusion 60, 056020. https://doi.org/10.1088/1741-4326/ab7fe1.

Peeters, A. G. 2000 Reduced charge state equations that describe Pfirsch Schlüter impurity transport in tokamak plasma. Phys. Plasmas 7, 268. https://doi.org/10.1063/1.873812.

Petrov, Y. V., PARKs, P. B. \& Harvey, R. W. 2021 Numerical simulation of the hot-tail runaway electron production mechanism using CQL3D and comparison with Smith-Verwichte analytical model. Plasma Phys. Control. Fusion 63, 035026. https://doi.org/10.1088/1361-6587/abdacc.

Reux, C., Paz-Soldan, C., Aleynikov, P., Bandaru, V., Ficker, O., Silburn, S., Hoelzl, M., Eidietis, N., LeHnen, M., SRIDHAR, S., et al. 2021 Demonstration of safe termination of megaampere relativistic electron beams in tokamaks. Phys. Rev. Lett. (accepted for publiction).

SÄrkimÄKi, K., Embreus, O., NARdon, E. \& FÜlÖP, T. \& JET CONTRIbUtors 2020 Assessing energy dependence of the transport of relativistic electrons in perturbed magnetic fields with orbit-following simulations. Nucl. Fusion 60, 126050. https://doi.org/10.1088/1741-4326/abb9e9.

SCHNEIDER, P. A. 2012 Characterization and scaling of the tokamak edge transport barrier. PhD thesis, Ludwig-Maximilians-Universität. https://doi.org/10.5282/edoc.14723.

Smith, H. M. \& Verwichte, E. 2008 Hot tail runaway electron generation in tokamak disruptions. Phys. Plasmas 15, 072502. https://doi.org/10.1063/1.2949692.

Stahl, A., Embréus, O., PAPP, G., LANDreman, M. \& FÜlÖP, T. 2016 Kinetic modelling of runaway electrons in dynamic scenarios. Nucl. Fusion 56, 112009. https://doi.org/10.1088/0029-5515/56/11/ 112009.

Summers, H. P. 2004 The ADAS User Manual, version 2.6. Available at: https://www.adas.ac.uk.

SVENNINGSSON, I. 2020 Hot-tail runaway electron generation in cooling fusion plasmas. Master's thesis, Chalmers University of Technology. Available at: https://hdl.handle.net/20.500.12380/300899. 
Svensson, P., Embreus, O., Newton, S. L., Särkimäki, K., Vallhagen, O. \& FülöP, T. 2021 Effects of magnetic perturbations and radiation on the runaway avalanche. J. Plasma Phys. 87, 905870207. https://doi.org/10.1017/S0022377820001592.

Tinguely, R. A., Granetz, R. S., Hoppe, M. \& Embréus, O. 2018 Measurements of runaway electron synchrotron spectra at high magnetic fields in Alcator C-Mod. Nucl. Fusion 58, 076019. https://doi.org/10.1088/1741-4326/aac444.

Vallhagen, O., Embreus, O., Pusztai, I., Hesslow, L. \& FÜlöP, T. 2020 Runaway dynamics in the DT phase of ITER operations in the presence of massive material injection. J. Plasma Phys. 86, 475860401. https://doi.org/10.1017/S0022377820000859. 